\title{
INTRODUCTION TO THE FUSION OF QUANTITATIVE AND QUALITATIVE BELIEFS
}

\author{
Jean DEZERT and Florentin SMARANDACHE
}

\begin{abstract}
The efficient management and combination of uncertain and conflicting sources of information remain of primal importance for the development of reliable information fusion systems. Advanced fusion systems must deal both with quantitative and qualitative aspects of beliefs expressed by the different sources of information (sensors, expert systems, human reports, etc). This paper introduces the theory of plausible and paradoxical reasoning, known as DSmT (DezertSmarandache Theory) in literature, developed originally for dealing with imprecise, uncertain and potentially highly conflicting sources of information providing quantitative beliefs on a given set of possible solutions of a given problem. We also propose in this paper new ideas on a possible extension of DSmT for the combination of uncertain and conflicting qualitative information in order to deal directly with beliefs expressed with linguistic labels instead of numerical values to be closer to the nature of information expressed in natural languages and available directly from human experts.
\end{abstract}

Keywords: Dezert-Smarandache Theory, DSmT, Information Fusion, Quantitative belief, Qualitative belief, Conflict management.

\section{Introduction}

The development of DSmT (Dezert-Smarandache Theory) [30] arises from the necessity to overcome the inherent limitations of DST (Dempster-Shafer Theory) [29] which are closely related with the acceptance of Shafer's model (i.e. working with an homogeneous frame of discernment $\Theta$ defined as a finite set of exhaustive and exclusive hypotheses $\theta_{i}, i=1, \ldots, n$ ), the third excluded middle principle, and Dempster's rule for the combination of independent sources of evidence. Limitations of DST are well reported in literature [46, 37, 47] and several alternative rules to Dempster's rule of combination can be found in $[10,42,16,18,28,30]$ and very recently in $[31,32,13]$. DSmT provides a new mathematical framework for the fusion of quantitative or qualitative beliefs which appears less restrictive and more general than the basis and constraints of DST.

INFORMATION \& SECURITY. An International Journal, Vol. 20, 2006, 7-45 
The basis of DSmT is the refutation of the principle of the third excluded middle and Shafer's model in general, since for a wide class of fusion problems the hypotheses one has to deal with can have different intrinsic natures and also appear only vague and imprecise in such a way that precise refinement is just impossible to obtain in reality so that the exclusive elements $\theta_{i}$ cannot be properly identified and defined. Many problems involving fuzzy/vague continuous and relative concepts described in natural language with different semantic contents and having no absolute interpretation enter in this category. Although DSmT was initially developed for the fusion of quantitative beliefs (i.e. numbers/masses in [0,1] satisfying a given set of constraints - see later), we will show in section 3 how it can be extended quite directly for the fusion of qualitative beliefs (i.e. when precise numbers are replaced by imprecise linguistic labels).

DSmT starts with the notion of free DSm model and considers $\Theta$ only as a frame of exhaustive elements which can potentially overlap and have different intrinsic natures and which also can change with time with new information and evidences received on the model itself. DSmT offers a flexibility on the structure of the model one has to deal with. When the free DSm model holds, the conjunctive consensus is used. If the free model does not fit the reality because it is known that some subsets of $\Theta$ contain elements truly exclusive but also possibly truly non existing at all at a given time (in dynamic $^{1}$ fusion), new fusion rules must be used to take into account these integrity constraints. The constraints can be explicitly introduced into the free DSm model to fit it adequately with our current knowledge of the reality; we actually construct a hybrid DSm model on which the combination will be efficiently performed. Shafer's model corresponds actually to a very specific hybrid DSm (and homogeneous) model including all possible exclusivity constraints. DSmT has been developed to work with any model and to combine imprecise, uncertain and potentially high conflicting sources for static and dynamic information fusion. DSmT refutes the idea that sources provide their (quantitative or qualitative) beliefs with the same absolute interpretation of elements of $\Theta$; what is considered as good for somebody can be considered as bad for somebody else. This paper is a revised and extended version of $[6,7,34,8]$.

After a short presentation of hyper-power set and DSm models in this section, we will present in section 2 the main combination rules for the fusion of quantitative precise or imprecise beliefs, i.e. the Classic (DSmC), the Hybrid DSm (DSmH) and the proportional conflict redistribution (PCR) rules of combination. Section 3 extends the quantitative fusion rules of section 2 to their qualitative counterparts. Such extension allows to deal directly with beliefs expressed with linguistic labels extracted from natural language.

\footnotetext{
${ }^{1}$ i.e. when the frame $\Theta$ and/or the model $\mathcal{M}$ is changing with time.
} 


\subsection{Notion of hyper-power set}

Let $\Theta=\left\{\theta_{1}, \ldots, \theta_{n}\right\}$ be a finite set (called frame) of $n$ exhaustive elements ${ }^{2}$. The free Dedekind's lattice denoted hyper-power set $D^{\Theta}$ [30] is defined as

1. $\emptyset, \theta_{1}, \ldots, \theta_{n} \in D^{\Theta}$.

2. If $A, B \in D^{\Theta}$, then $A \cap B$ and $A \cup B$ belong to $D^{\Theta}$.

3. No other elements belong to $D^{\Theta}$, except those obtained by using rules 1 or 2 .

If $|\Theta|=n$, then $\left|D^{\Theta}\right| \leq 2^{2^{n}}$. The generation of $D^{\Theta}$ is presented in [30]. Since for any given finite set $\Theta,\left|D^{\Theta}\right| \geq\left|2^{\Theta}\right|$, we call $D^{\Theta}$ the hyper-power set of $\Theta$. $\left|D^{\Theta}\right|$ for $n \geq 1$ follows the sequence of Dedekind's numbers:1,2,5,19,167,... An analytical expression of Dedekind's numbers obtained by Tombak and al. can be found in [30].

Example: If $\Theta=\left\{\theta_{1}, \theta_{2}, \theta_{3}\right\}$, then its hyper-power set $D^{\Theta}$ includes the following nineteen elements: $\emptyset, \theta_{1} \cap \theta_{2} \cap \theta_{3}, \theta_{1} \cap \theta_{2}, \theta_{1} \cap \theta_{3}, \theta_{2} \cap \theta_{3},\left(\theta_{1} \cup \theta_{2}\right) \cap \theta_{3},\left(\theta_{1} \cup \theta_{3}\right) \cap \theta_{2}$, $\left(\theta_{2} \cup \theta_{3}\right) \cap \theta_{1},\left(\theta_{1} \cap \theta_{2}\right) \cup\left(\theta_{1} \cap \theta_{3}\right) \cup\left(\theta_{2} \cap \theta_{3}\right), \theta_{1}, \theta_{2}, \theta_{3},\left(\theta_{1} \cap \theta_{2}\right) \cup \theta_{3},\left(\theta_{1} \cap \theta_{3}\right) \cup \theta_{2}$, $\left(\theta_{2} \cap \theta_{3}\right) \cup \theta_{1}, \theta_{1} \cup \theta_{2}, \theta_{1} \cup \theta_{3}, \theta_{2} \cup \theta_{3}$ and $\theta_{1} \cup \theta_{2} \cup \theta_{3}$.

\subsection{Free and hybrid DSm models}

$\Theta=\left\{\theta_{1}, \ldots, \theta_{n}\right\}$ denotes the finite set of hypotheses characterizing the fusion problem. $D^{\Theta}$ constitutes the free DSm model $\mathcal{M}^{f}(\Theta)$ and allows to work with fuzzy concepts which depict a continuous and relative intrinsic nature. Such kinds of concepts cannot be precisely refined with an absolute interpretation because of the unapproachable universal truth. When all $\theta_{i}$ are truly exclusive discrete elements, $D^{\Theta}$ reduces to the classical power set $2^{\Theta}$. This is what we call the Shafer's model, denoted $\mathcal{M}^{0}(\Theta)$. Between the free DSm model and the Shafer's model, there exists a wide class of fusion problems represented in term of DSm hybrid models where $\Theta$ involves both fuzzy continuous concepts and discrete hypotheses. In such class, some exclusivity constraints and possibly some non-existential constraints (especially when working on dynamic fusion) have to be taken into account. Each hybrid fusion problem is then characterized by a proper hybrid DSm model $\mathcal{M}(\Theta)$ with $\mathcal{M}(\Theta) \neq \mathcal{M}^{f}(\Theta)$ and $\mathcal{M}(\Theta) \neq \mathcal{M}^{0}(\Theta)$. The main differences between DST and DSmT are (1) the model on which one works with, and (2) the choice of the combination rule. We use here the generic notation $G$ for denoting either $D^{\Theta}$ (when working in DSmT) or $2^{\Theta}$ (when working in DST). We denote $G^{*}$ the set $G$ from which the empty set is excluded $\left.\left(G^{*}=G \backslash\{\emptyset)\right\}\right)$.

\footnotetext{
${ }^{2}$ We do not assume here that elements $\theta_{i}$ have the same intrinsic nature and are necessary exclusive. There is no restriction on $\theta_{i}$ but the exhaustivity which is not a strong constraint since we can always introduce if necessary a closure element representing all missing hypotheses, say $\theta_{0}$, in order to always work in a closed world.
} 
- A 3D Example of free DSm model: When $\Theta=\left\{\theta_{1}, \theta_{2}, \theta_{3}\right\}$, the free-model $\mathcal{M}^{f}(\Theta)$ corresponds to the following Venn diagram where all elements can overlap partially but with vague boundaries in such a way that no exact/precise refinement is possible.

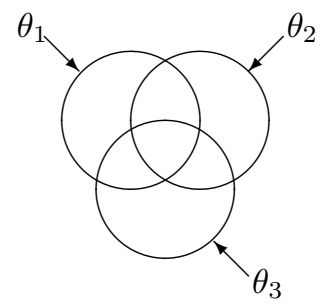

Figure 1: Venn Diagram for the free DSm model $\mathcal{M}^{f}(\Theta)$

- A 3D Example of a hybrid DSm model: Let's consider $\Theta=\left\{\theta_{1}, \theta_{2}, \theta_{3}\right\}$ and only the exclusivity constraint of $\theta_{3}$ with respect to $\theta_{1}$ and $\theta_{2}$, then one gets (see figure 2) the following Venn diagram for this specific hybrid DSm model $\mathcal{M}(\Theta)$ defined by $\Theta$ and the chosen (integrity) constraint.

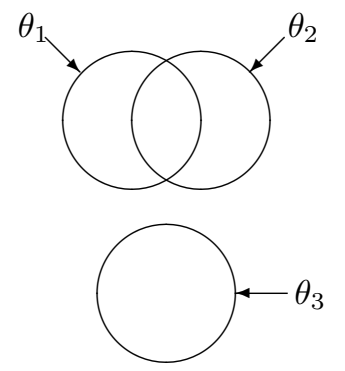

Figure 2: Venn Diagram for a hybrid DSm model $\mathcal{M}(\Theta)$

- A 3D Example of Shafer's model: Let's consider $\Theta=\left\{\theta_{1}, \theta_{2}, \theta_{3}\right\}$. Shafer's model, denoted $\mathcal{M}^{0}(\Theta)$ assumes all elements of $\Theta$ being truly exhaustive and exclusive. Its corresponding Venn diagram corresponds to following figure. 


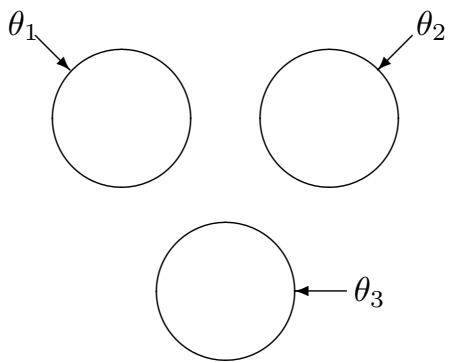

Figure 3: Venn Diagram for Shafer's model $\mathcal{M}^{0}(\Theta)$

\section{Fusion of quantitative beliefs}

\subsection{Quantitative belief functions}

In DSmT framework, a (precise) quantitative basic belief assignment ${ }^{3}$ (bba) associated with a given source of information (body of evidence) about a frame $\Theta$ is defined as a precise mapping $m($.$) from G$ into $[0,1]$, i.e. $m():. G \rightarrow[0,1]$ satisfying:

$$
m(\emptyset)=0 \quad \text { and } \quad \sum_{A \in G} m(A)=1
$$

From $m($.$) , we define the (quantitative) credibility and plausibility functions as:$

$$
\operatorname{Bel}(A) \triangleq \sum_{\substack{B \subseteq A \\ B \in G}} m(B) \quad \text { and } \quad \operatorname{Pl}(A) \triangleq \sum_{\substack{B \cap A \neq \emptyset \\ B \in G}} m(B)
$$

These definitions remain compatible with the definitions of $\operatorname{Bel}($.$) and \mathrm{Pl}($.$) given in$ DST when $\mathcal{M}^{0}(\Theta)$ holds [29] since in that case $G=D^{\Theta}$ reduces to classical powerset $2^{\Theta}$.

\subsection{Combinations of precise quantitative beliefs}

We present here the three main DSm fusion rules proposed in DSmT framework for the combination of precise quantitative beliefs. The most simple rule is the Classic DSm rule (DSmC) which corresponds to the consensus operator on hyper-power set when the free DSm model holds. The second and more sophisticated one is the DSm hybrid rule (DSmH) [30] which allows to work on any static or dynamic hybrid model and also to work on the Shafer's model whenever this model holds. (DSmH) is a direct extension of Dubois \& Prade's rule [10] for dealing with the dynamic/temporal fusion (i.e. when the frame and its model/constraints change with time). Then we present

\footnotetext{
${ }^{3}$ also called belief mass in the literature
} 
the proportional conflict redistribution rule \#5 (PCR5) which proposes a more subtle transfer of the conflicting masses than (DSmH) [32, 31]. (DSmH) and PCR rules are mathematically well defined and work both with any models and whatever the value the degree of conflict can take. In practice, when reliabilities of sources are known, we can easily take them into account in all DSm-based fusion rules by discounting them by the proper discounting factor and using classical discounting approach of beliefs [29, 30]. We will not go deeper in the presentation of well-known discounting techniques here since we consider them less fundamental than the combination. We just want to emphasize here that this preprocessing/discounting step, although very important from practical point of view must however never appear as a substitute or as an artificial engineering trick to circumvent the inherent deficiencies of a chosen combination rule. Even if the DSm-based rules work for any degree of conflict between sources, we do not claim that they should be applied blindly in practice when conflict becomes very large, without trying first to analyze the origins of the partial conflicts, estimate and take into account (when it is possible) the reliability of each source before their combination. But once all these necessary preliminary works (deep analysis of the problems, the refinement of the model, and reliability assessment of each source) have been done, one has always to choose what we consider the most legitimate combination rule we will apply. DSm-based rule provide possible new solutions and serious alternatives for the combination of uncertain, imprecise and conflicting information. Comparisons of the different main quantitative rules of combination with several examples can be found in $[30,32,31,8,13]$.

\section{Classic DSm fusion rule (DSmC)}

When the free DSm model $\mathcal{M}^{f}(\Theta)$ holds, the conjunctive consensus, called DSm classic rule (DSmC), is performed on $D^{\Theta}$. DSmC of two independent ${ }^{4}$ sources associated with gbba $m_{1}($.$) and m_{2}($.$) is thus given \forall C \in D^{\Theta}$ by [30]:

$$
m_{D S m C}(C)=\sum_{\substack{A, B \in D^{\Theta} \\ A \cap B=C}} m_{1}(A) m_{2}(B)
$$

$D^{\Theta}$ being closed under $\cup$ and $\cap$ operators, DSmC guarantees that $m($.$) is a proper$ gbba. DSmC is commutative and associative and can be used for the fusion of sources involving fuzzy concepts whenever $\mathcal{M}^{f}(\Theta)$ holds. It can be easily extended for the fusion of $k>2$ independent sources [30].

\footnotetext{
${ }^{4}$ While independence is a difficult concept to define in all theories managing epistemic uncertainty, we consider that two sources of evidence are independent (i.e. distinct and noninteracting) if each leaves one totally ignorant about the particular value the other will take.
} 


\section{Example for (DSmC)}

Let's consider a generalization of Zadeh's example [46, 47] and take $\Theta=\left\{\theta_{1}, \theta_{2}, \theta_{3}\right\}$, $0<\epsilon_{1}, \epsilon_{2}<1$, be two positive numbers and two experts providing the quantitative and precise bba $m_{1}\left(\theta_{1}\right)=1-\epsilon_{1}, m_{1}\left(\theta_{2}\right)=0, m_{1}\left(\theta_{3}\right)=\epsilon_{1}, m_{2}\left(\theta_{1}\right)=0, m_{2}\left(\theta_{2}\right)=1-\epsilon_{2}$ and $m_{2}\left(\theta_{3}\right)=\epsilon_{2}$.

If one adopts the free-DSm model for $\Theta$ (i.e. we accept the non exclusivity of hypotheses), using (DSmC) one gets zero for all masses of $D^{\Theta}$ except the following ones:

$$
\begin{gathered}
m_{D S m C}\left(\theta_{3}\right)=\epsilon_{1} \epsilon_{2} \\
m_{D S m C}\left(\theta_{1} \cap \theta_{2}\right)=\left(1-\epsilon_{1}\right)\left(1-\epsilon_{2}\right) \\
m_{D S m C}\left(\theta_{1} \cap \theta_{3}\right)=\left(1-\epsilon_{1}\right) \epsilon_{2} \\
m_{D S m C}\left(\theta_{2} \cap \theta_{3}\right)=\left(1-\epsilon_{2}\right) \epsilon_{1}
\end{gathered}
$$

\section{Hybrid DSm fusion rule (DSmH)}

When $\mathcal{M}^{f}(\Theta)$ does not hold (some integrity constraints exist), one deals with a proper DSm hybrid model $\mathcal{M}(\Theta) \neq \mathcal{M}^{f}(\Theta)$. DSm hybrid rule (DSmH) for $k \geq 2$ independent sources is thus defined for all $A \in D^{\Theta}$ as [30]:

$$
m_{D S m H}(A) \triangleq \phi(A) \cdot\left[S_{1}(A)+S_{2}(A)+S_{3}(A)\right]
$$

where $\phi(A)$ is the characteristic non-emptiness function of a set $A$, i.e. $\phi(A)=1$ if $A \notin \emptyset$ and $\phi(A)=0$ otherwise, where $\emptyset \triangleq\left\{\emptyset_{\mathcal{M}}, \emptyset\right\}$. $\emptyset_{\mathcal{M}}$ is the set of all elements of $D^{\Theta}$ which have been forced to be empty through the constraints of the model $\mathcal{M}$ and $\emptyset$ is the classical/universal empty set. $S_{1}(A) \equiv m_{\mathcal{M}^{f}(\theta)}(A), S_{2}(A), S_{3}(A)$ are defined by

$$
\begin{aligned}
& S_{1}(A) \triangleq \sum_{\substack{X_{1}, X_{2}, \ldots, X_{k} \in D^{\Theta} \\
\left(X_{1} \cap X_{2} \cap \ldots \cap X_{k}\right)=A}} \prod_{i=1}^{k} m_{i}\left(X_{i}\right) \\
& S_{2}(A) \triangleq \sum_{\substack{X_{1}, X_{2}, \ldots, X_{k} \in \emptyset \\
[\mathcal{U}=A] \vee\left[(\mathcal{U} \in \emptyset) \wedge\left(A=I_{t}\right)\right]}} \prod_{i=1}^{k} m_{i}\left(X_{i}\right) \\
& S_{3}(A) \triangleq \sum_{\substack{\left.X_{1}, X_{2}, \ldots, X_{k} \in D^{\Theta}\right) \\
u\left(c\left(X_{1} \cap X_{2} \cap \ldots \cap X_{k}\right)\right)=A \\
\left(X_{1} \cap X_{2} \cap \ldots \cap X_{k}\right) \in \emptyset}} \prod_{i=1}^{k} m_{i}\left(X_{i}\right)
\end{aligned}
$$


with $\mathcal{U} \triangleq u\left(X_{1}\right) \cup \ldots \cup u\left(X_{k}\right)$ where $u(X)$ is the union of all $\theta_{i}$ that compose $X$, $I_{t} \triangleq \theta_{1} \cup \ldots \cup \theta_{n}$ is the total ignorance, and $c(X)$ is the canonical form ${ }^{5}$ of $X$, i.e. its simplest form (for example if $X=(A \cap B) \cap(A \cup B \cup C), c(X)=A \cap B) . S_{1}(A)$ is nothing but the DSmC rule for $k$ independent sources based on $\mathcal{M}^{f}(\Theta) ; S_{2}(A)$ is the mass of all relatively and absolutely empty sets which is transferred to the total or relative ignorances associated with non existential constraints (if any, like in some dynamic problems); $S_{3}(A)$ transfers the sum of relatively empty sets directly onto the canonical disjunctive form of non-empty sets. DSmH generalizes DSmC and allows to work on Shafer's model. It is definitely not equivalent to Dempster's rule since these rules are different. DSmH works for any models (free DSm model, Shafer's model or any hybrid models) when manipulating precise bba. A recent report on DSmT including MatLab ${ }^{6}$ codes can be found in [14].

\section{Example for (DSmH)}

Let's consider the previous example with $\Theta=\left\{\theta_{1}, \theta_{2}, \theta_{3}\right\}, 0<\epsilon_{1}, \epsilon_{2}<1$, be two positive numbers and two experts providing the quantitative and precise bba $m_{1}\left(\theta_{1}\right)=$ $1-\epsilon_{1}, m_{1}\left(\theta_{2}\right)=0, m_{1}\left(\theta_{3}\right)=\epsilon_{1}, m_{2}\left(\theta_{1}\right)=0, m_{2}\left(\theta_{2}\right)=1-\epsilon_{2}$ and $m_{2}\left(\theta_{3}\right)=\epsilon_{2}$ and now assume that Shafer's model holds, i.e. we assume that $\theta_{1}, \theta_{2}$ and $\theta_{3}$ are truly exclusive.

- based on (DSmH) fusion rule (4), on gets:

$$
\begin{gathered}
m_{D S m H}\left(\theta_{3}\right)=\epsilon_{1} \epsilon_{2} \\
m_{D S m H}\left(\theta_{1} \cup \theta_{2}\right)=\left(1-\epsilon_{1}\right)\left(1-\epsilon_{2}\right) \\
m_{D S m H}\left(\theta_{1} \cup \theta_{3}\right)=\left(1-\epsilon_{1}\right) \epsilon_{2} \\
m_{D S m H}\left(\theta_{2} \cup \theta_{3}\right)=\left(1-\epsilon_{2}\right) \epsilon_{1}
\end{gathered}
$$

All other masses are zero. This result makes sense since it depends truly on the values of $\epsilon_{1}$ and $\epsilon_{2}$ contrariwise to Dempster's rule according next item.

- using Dempster-Shafer's (DS) rule of combination [29], one gets

$$
m_{D S}\left(\theta_{3}\right)=\frac{\left(\epsilon_{1} \epsilon_{2}\right)}{\left(1-\epsilon_{1}\right) \cdot 0+0 \cdot\left(1-\epsilon_{2}\right)+\epsilon_{1} \epsilon_{2}}=1
$$

\footnotetext{
${ }^{5}$ The canonical form is introduced here explicitly in order to improve the original formula given in [30] for preserving the neutral impact of the vacuous belief mass $m(\Theta)=1$ within complex hybrid models. Actually all propositions involved in formulas are expressed in their canonical form, i.e. conjunctive normal form, also known as conjunction of disjunctions in Boolean algebra, which is unique.

${ }^{6}$ MatLab is a trademark of The MathWorks, Inc., U.S.A.
} 
which is absurd (or at least counter-intuitive). Note that whatever positive values for $\epsilon_{1}, \epsilon_{2}$ are, Dempster's rule gives always the same result (one) which is abnormal. The only acceptable and correct result obtained by Dempster's rule is really obtained only in the trivial case when $\epsilon_{1}=\epsilon_{2}=1$, i.e. when both sources agree in $\theta_{3}$ with certainty which is obvious.

When $\epsilon_{1}=\epsilon_{2}=1 / 2$, one otains

$$
\begin{array}{lll}
m_{1}\left(\theta_{1}\right)=1 / 2 & m_{1}\left(\theta_{2}\right)=0 & m_{1}\left(\theta_{3}\right)=1 / 2 \\
m_{2}\left(\theta_{1}\right)=0 & m_{2}\left(\theta_{2}\right)=1 / 2 & m_{2}\left(\theta_{3}\right)=1 / 2
\end{array}
$$

Dempster's rule still yields $m_{D S}\left(\theta_{3}\right)=1$ while DSmH based on the same Shafer's model yields now $m_{D S m H}\left(\theta_{3}\right)=1 / 4, m_{D S m H}\left(\theta_{1} \cup \theta_{2}\right)=1 / 4, m_{D S m H}\left(\theta_{1} \cup \theta_{3}\right)=$ $1 / 4, m_{D S m H}\left(\theta_{2} \cup \theta_{3}\right)=1 / 4$ which is more acceptable upon authors opinion. A detailed discussion on this example (and on more examples) with answers to recent criticisms published in [15] can be found in [8].

\section{Proportional Conflict Redistribution rule no 5 (PCR5)}

Instead of applying a direct transfer of partial conflicts onto partial uncertainties as with (DSmH), the idea behind the Proportional Conflict Redistribution (PCR) rule [31, 32] is to transfer (total or partial) conflicting masses to non-empty sets involved in the conflicts proportionally with respect to the masses assigned to them by sources as follows:

1. calculation the conjunctive rule of the belief masses of sources;

2. calculation the total or partial conflicting masses;

3. redistribution of the (total or partial) conflicting masses to the non-empty sets involved in the conflicts proportionally with respect to their masses assigned by the sources.

The way the conflicting mass is redistributed yields actually several versions of PCR rules. These PCR fusion rules work for any degree of conflict, for any DSm models (Shafer's model, free DSm model or any hybrid DSm model) and both in DST and DSmT frameworks for static or dynamical fusion situations. We present here the most achieved proportional conflict redistribution rule (rule no 5) denoted (PCR5) in $[31,32]$. PCR5 is what we think the most efficient PCR fusion rule for the combination of two sources. A more intuitive version of PCR5 for $s>=3$ sources and denoted PCR6 has been recently proposed by Martin and Osswald in [19]. (PCR6) coincides with (PCR5) for the two-source case, but differs from (PCR5) when combining altogether more than two sources. 
PCR5 rule redistributes the partial conflicting mass to the elements involved in the partial conflict, considering the conjunctive normal form of the partial conflict. PCR5 is what we think the most interesting redistribution of conflicting mass to non-empty sets following the logic of the conjunctive rule. (PCR5) does a better redistribution of the conflicting mass than Dempster's rule since (PCR5) goes backwards on the tracks of the conjunctive rule and redistributes the conflicting mass only to the sets involved in the conflict and proportionally to their masses put in the conflict. (PCR5) rule is quasi-associative and preserves the neutral impact of the vacuous belief assignment because in any partial conflict, as well in the total conflict (which is a sum of all partial conflicts), the conjunctive normal form of each partial conflict does not include $\Theta$ since $\Theta$ is a neutral element for intersection (conflict), therefore $\Theta$ gets no mass after the redistribution of the conflicting mass. We have proved in [31] the continuity property of the (PCR5) result with continuous variations of bba to combine. The general (PCR5) formula for $s \geq 2$ sources is given by [31] $m_{P C R 5}(\emptyset)=0$ and $\forall X \in G \backslash\{\emptyset\}$

$$
\begin{aligned}
& m_{P C R 5}(X)=m_{12 \ldots s}(X)+\sum_{2 \leq t \leq s} \sum_{X_{j_{2}}, \ldots, X_{j_{t}} \in G \backslash\{X\}}
\end{aligned}
$$

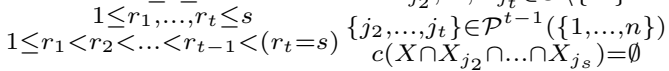

$$
\begin{aligned}
& \left\{i_{1}, \ldots, i_{s}\right\} \in \mathcal{P}^{s}(\{1, \ldots, s\}) \\
& \frac{\left(\prod_{k_{1}=1}^{r_{1}} m_{i_{k_{1}}}(X)^{2}\right) \cdot\left[\prod_{l=2}^{t}\left(\prod_{k_{l}=r_{l-1}+1}^{r_{l}} m_{i_{k_{l}}}\left(X_{j_{l}}\right)\right]\right.}{\left(\prod_{k_{1}=1}^{r_{1}} m_{i_{k_{1}}}(X)\right)+\left[\sum_{l=2}^{t}\left(\prod_{k_{l}=r_{l-1}+1}^{r_{l}} m_{i_{k_{l}}}\left(X_{j_{l}}\right)\right]\right.}
\end{aligned}
$$

where $G$ corresponds to classical power-set $2^{\Theta}$ if Shafer's model is used or $G$ corresponds to a constrained hyper-power set $D^{\Theta}$ if any other hybrid DSm model is used instead; $i, j, k, r, s$ and $t$ in (8) are integers.

$$
m_{12 \ldots s}(X) \equiv m_{\cap}(X)=\sum_{\substack{X_{1}, \ldots, X_{s} \in G \\ X_{1} \cap \ldots \cap X_{s}=X}} \prod_{i=1}^{s} m_{i}\left(X_{i}\right)
$$

corresponds to the conjunctive consensus on $X$ between $s$ sources and where all denominators are different from zero. If a denominator is zero, that fraction is discarded; the set of all subsets of $k$ elements from $\{1,2, \ldots, n\}$ (permutations of $n$ elements taken by $k$ ) was denoted $\mathcal{P}^{k}(\{1,2, \ldots, n\})$, the order of elements doesn't count. $c(X)$ is the canonical form (conjunctive normal form) of $X$.

When $s=2$ (fusion of only two sources), the previous (PCR5) formula reduces to its simple following fusion formula: $m_{P C R 5}(\emptyset)=0$ and $\forall X \in G \backslash\{\emptyset\}$

$$
m_{P C R 5}(X)=m_{12}(X)+\sum_{\substack{Y \in G \backslash\{X\} \\ c(X \cap Y)=\emptyset}}\left[\frac{m_{1}(X)^{2} m_{2}(Y)}{m_{1}(X)+m_{2}(Y)}+\frac{m_{2}(X)^{2} m_{1}(Y)}{m_{2}(X)+m_{1}(Y)}\right]
$$


For $\Theta=\left\{\theta_{1}, \theta_{2}, \ldots, \theta_{n}\right\}$ with Shafer's model and $s=2$ Bayesian equi-reliable sources, i.e. when quantitative bba $m_{1}($.$) and m_{2}($.$) reduce to subjective probability$ measures $P_{1}($.$) and P_{2}($.$) , it can be shown [31] after elementary algebraic derivations$ that previous (PCR5) formula reduces to the following simple formula, $P_{12}^{P C R 5}(\emptyset)=0$ and $\forall \theta_{i} \in \Theta$,

$$
\begin{aligned}
P_{12}^{P C R 5}\left(\theta_{i}\right) & =P_{1}\left(\theta_{i}\right) \sum_{j=1}^{n} \frac{P_{1}\left(\theta_{i}\right) P_{2}\left(\theta_{j}\right)}{P_{1}\left(\theta_{i}\right)+P_{2}\left(\theta_{j}\right)}+P_{2}\left(\theta_{i}\right) \sum_{j=1}^{n} \frac{P_{2}\left(\theta_{i}\right) P_{1}\left(\theta_{j}\right)}{P_{2}\left(\theta_{i}\right)+P_{1}\left(\theta_{j}\right)} \\
& =\sum_{s=1,2} P_{s}\left(\theta_{i}\right)\left[\sum_{j=1}^{n} \frac{P_{s}\left(\theta_{i}\right) P_{s^{\prime} \neq s}\left(\theta_{j}\right)}{P_{s}\left(\theta_{i}\right)+P_{s^{\prime} \neq s}\left(\theta_{j}\right)}\right]
\end{aligned}
$$

It can be checked moreover that $P_{12}^{P C R 5}($.$) defines a subjective-combined proba-$ bility measure satisfying all axioms of classical Probability Theory.

\section{Examples for (PCR5)}

- Example 1: Let's take $\Theta=\{A, B\}$ of exclusive elements (Shafer's model), and the following bba:

\begin{tabular}{|c|ccc|}
\hline & $A$ & $B$ & $A \cup B$ \\
\hline$m_{1}()$. & 0.6 & 0 & 0.4 \\
\hline$m_{2}()$. & 0 & 0.3 & 0.7 \\
\hline \hline$m_{\cap}()$. & 0.42 & 0.12 & 0.28 \\
\hline
\end{tabular}

The conflicting mass is $k_{12}=m_{\cap}(A \cap B)=m_{1}(A) m_{2}(B)+m_{1}(B) m_{2}(A)=$ 0.18 . Therefore $A$ and $B$ are the only focal ${ }^{7}$ elements involved in the conflict. Hence according to the (PCR5) hypothesis only $A$ and $B$ deserve a part of the conflicting mass and $A \cup B$ does not deserve. With (PCR5), one redistributes the conflicting mass $k_{12}=0.18$ to $A$ and $B$ proportionally with the masses $m_{1}(A)$ and $m_{2}(B)$ assigned to $A$ and $B$ respectively. Let $x$ be the conflicting mass to be redistributed to $A$, and $y$ the conflicting mass redistributed to $B$, then

$$
\frac{x}{0.6}=\frac{y}{0.3}=\frac{x+y}{0.6+0.3}=\frac{0.18}{0.9}=0.2
$$

hence $x=0.6 \cdot 0.2=0.12, y=0.3 \cdot 0.2=0.06$. Thus, the final result using the (PCR5) rule is

$$
\left\{\begin{array}{l}
m_{P C R 5}(A)=0.42+0.12=0.54 \\
m_{P C R 5}(B)=0.12+0.06=0.18 \\
m_{P C R 5}(A \cup B)=0.28
\end{array}\right.
$$

${ }^{7} \mathrm{a}$ focal element is an element carrying strictly positive belief mass. 
For comparison, here are the results obtained from Dempster's rule (DS), (DSmH) and (PCR5):

\begin{tabular}{|l||ccc|}
\hline & $A$ & $B$ & $A \cup B$ \\
\hline$m_{D S}$ & 0.512 & 0.146 & 0.342 \\
$m_{D S m H}$ & 0.420 & 0.120 & 0.460 \\
$m_{P C R 5}$ & 0.540 & 0.180 & 0.280 \\
\hline
\end{tabular}

- Example 2: Let's modify example 1 and consider

\begin{tabular}{|c|ccc|}
\hline & $A$ & $B$ & $A \cup B$ \\
\hline$m_{1}()$. & 0.6 & 0 & 0.4 \\
\hline$m_{2}()$. & 0.2 & 0.3 & 0.5 \\
\hline \hline$m_{\cap}()$. & 0.50 & 0.12 & 0.20 \\
\hline
\end{tabular}

The conflicting mass $k_{12}=m_{\cap}(A \cap B)$ as well as the distribution coefficients for the (PCR5) remains the same as in the previous example but one gets now

\begin{tabular}{|l||ccc|}
\hline & $A$ & $B$ & $A \cup B$ \\
\hline$m_{D S}$ & 0.609 & 0.146 & 0.231 \\
$m_{D S m H}$ & 0.500 & 0.120 & 0.380 \\
$m_{P C R 5}$ & 0.620 & 0.180 & 0.200 \\
\hline
\end{tabular}

- Example 3: Let's modify example 2 and consider

\begin{tabular}{|c|ccc|}
\hline & $A$ & $B$ & $A \cup B$ \\
\hline$m_{1}()$. & 0.6 & 0.3 & 0.1 \\
\hline$m_{2}()$. & 0.2 & 0.3 & 0.5 \\
\hline \hline$m_{\cap}()$. & 0.44 & 0.27 & 0.05 \\
\hline
\end{tabular}

The conflicting mass $k_{12}=0.24=m_{1}(A) m_{2}(B)+m_{1}(B) m_{2}(A)=0.24$ is now different from previous examples, which means that $m_{2}(A)=0.2$ and $m_{1}(B)=0.3$ did make an impact on the conflict. Therefore $A$ and $B$ are the only focal elements involved in the conflict and thus only $A$ and $B$ deserve a part of the conflicting mass. (PCR5) redistributes the partial conflicting mass 0.18 to $A$ and $B$ proportionally with the masses $m_{1}(A)$ and $m_{2}(B)$ and also the partial conflicting mass 0.06 to $A$ and $B$ proportionally with the masses $m_{2}(A)$ and $m_{1}(B)$. After all derivations (see [13] for details), one finally gets

\begin{tabular}{|l||ccc|}
\hline & $A$ & $B$ & $A \cup B$ \\
\hline$m_{D S}$ & 0.579 & 0.355 & 0.066 \\
$m_{D S m H}$ & 0.440 & 0.270 & 0.290 \\
$m_{P C R 5}$ & 0.584 & 0.366 & 0.050 \\
\hline
\end{tabular}


One clearly sees that $m_{D S}(A \cup B)$ gets some mass from the conflicting mass although $A \cup B$ does not deserve any part of the conflicting mass (according to (PCR5) hypothesis) since $A \cup B$ is not involved in the conflict (only $A$ and $B$ are involved in the conflicting mass). Dempster's rule appears to us less exact than (PCR5) and Inagaki's rules [16]. It can be showed [13] that Inagaki's fusion rule [16] (with an optimal choice of tuning parameters) can become in some cases very close to (PCR5) but upon our opinion (PCR5) result is more exact (at least less ad-hoc than Inagaki's one ).

- Example 4: Zadeh's example [46, 47]

Let's consider $\Theta=\{M, C, T\}$ as the frame of three potential origins about possible diseases of a patient ( $M$ standing for meningitis, $C$ for concussion and $T$ for tumor), the Shafer's model and the two following belief assignments provided by two independent doctors after examination of the same patient.

$$
\begin{array}{lll}
m_{1}(M)=0.9 & m_{1}(C)=0 & m_{1}(T)=0.1 \\
m_{2}(M)=0 & m_{2}(C)=0.9 & m_{2}(T)=0.1
\end{array}
$$

The total conflicting mass is high since it is

$$
m_{1}(M) m_{2}(C)+m_{1}(M) m_{2}(T)+m_{2}(C) m_{1}(T)=0.99
$$

- with Dempster's rule and Shafer's model (DS), one gets the counter-intuitive result (see justifications in $[46,10,42,37,30]$ and criticism against them in [15]): $m_{D S}(T)=1$

- with Yager's rule ${ }^{8}$ [42] and Shafer's model: $m_{Y}(M \cup C \cup T)=0.99$ and $m_{Y}(T)=0.01$

- with (DSmH) and Shafer's model:

$$
\begin{gathered}
m_{D S m H}(M \cup C)=0.81 \quad m_{D S m H}(T)=0.01 \\
m_{D S m H}(M \cup T)=m_{D S m H}(C \cup T)=0.09
\end{gathered}
$$

- The Dubois \& Prade's rule (DP) [10] based on Shafer's model provides in Zadeh's example the same result as (DSmH), because (DP) and (DSmH) coincide in all static fusion problems ${ }^{9}$.

- with (PCR5) and Shafer's model:

$$
m_{P C R 5}(M)=m_{P C R 5}(C)=0.486 \quad m_{P C R 5}(T)=0.028
$$

\footnotetext{
${ }^{8}$ Ronald Yager suggested in his rule to transfer the total conflicting mass to the total ignorance instead using normalization as with Dempster's rule.

${ }^{9}$ Indeed (DP) rule has been developed for static fusion only while (DSmH) has been developed to take into account the possible dynamicity of the frame itself and also its associated model.
} 
One sees that when the total conflict between sources becomes high, DSmT is able (upon authors opinion) to manage more adequately through either (DSmH) or (PCR5) rules the combination of information than Dempster's rule, even when working with Shafer's model - which is only a specific hybrid model. (DSmH) rule is in agreement with (DP) rule for the static fusion, but (DSmH) and (DP) rules differ in general (for non degenerate cases) for dynamic fusion while (PCR5) rule seems more exact because of the proper proportional conflict redistribution of partial conflicts only to elements involved in the partial conflicts. Besides this particular example, we showed in $[30,31]$ that there exist several infinite classes of counter-examples to Dempster's rule which can be solved by DSmT.

\subsection{Combination of imprecise quantitative beliefs}

When sources are unable to provide precise quantitative basic beliefs assignments (bba) $m($.$) , they can in some cases at least express their quantitative belief assignment on a$ frame $\Theta$ in an imprecise manner as admissible imprecise quantitative basic beliefs assignments $m^{I}($.$) whose values are real subunitary intervals of [0,1]$, or even more general as real subunitary sets (i.e. sets, not necessarily intervals). In the general case, these sets can be unions of (closed, open, or half-open/half-closed) intervals and/or scalars all in $[0,1]$.

\section{Definition of imprecise quantitative basic beliefs assignment}

An imprecise quantitative bba $m^{I}($.$) is mathematically defined as m^{I}():. D^{\Theta} \rightarrow$ $\mathcal{P}([0,1]) \backslash\{\emptyset\}$ where $\mathcal{P}([0,1])$ is the set of all subsets of the interval $[0,1] . m^{I}($.$) over$ $D^{\Theta}$ is said admissible if and only if there exists for every $X \in D^{\Theta}$ at least one real number $m(X) \in m^{I}(X)$ such that $\sum_{X \in D^{\Theta}} m(X)=1 . m^{I}($.$) is a normal extension$ of $m($.$) from scalar values to set values. For example, if a source m($.$) is not sure about$ a scalar value $m(A)=0.3$, it may be considered an imprecise source which gives a set value say $m^{I}(A)=[0.2,0.4]$.

\section{Operators on sets}

The following simple commutative operators on sets (addition $\boxplus$ and multiplication $\odot$ ) are required [30] for fusion of imprecise bba:

- Addition :

$$
\mathcal{X}_{1} \boxplus \mathcal{X}_{2} \triangleq\left\{x \mid x=x_{1}+x_{2}, x_{1} \in \mathcal{X}_{1}, x_{2} \in \mathcal{X}_{2}\right\}
$$

- Multiplication :

$$
\mathcal{X}_{1} \boxminus \mathcal{X}_{2} \triangleq\left\{x \mid x=x_{1} \cdot x_{2}, x_{1} \in \mathcal{X}_{1}, x_{2} \in \mathcal{X}_{2}\right\}
$$


These operators are generalized for the summation and products of $n \geq 2$ sets as follows

$$
\begin{gathered}
\sum_{k=1, \ldots, n} \mathcal{X}_{k}=\left\{x \mid x=\sum_{k=1, \ldots, n} x_{k}, x_{1} \in \mathcal{X}_{1}, \ldots, x_{n} \in \mathcal{X}_{n}\right\} \\
\prod_{k=1, \ldots, n} \mathcal{X}_{k}=\left\{x \mid x=\prod_{k=1, \ldots, n} x_{k}, x_{1} \in \mathcal{X}_{1}, \ldots, x_{n} \in \mathcal{X}_{n}\right\}
\end{gathered}
$$

From these operators, one easily generalizes (DSmC) and (DSmH) fusion rules from scalars to sets ([30] chap. 6) to obtain their imprecise counterparts. In order to extend (PCR5) to its imprecise counterpart, i.e. (imp-PCR5) fusion rule, for dealing with imprecise quantitative belief assignments, we need also to introduce the division operator on sets as follows:

- Division (for the case when $0 \notin \mathcal{X}_{2}, \inf \left(\mathcal{X}_{2}\right) \neq 0$ and $\sup \left(\mathcal{X}_{2}\right) \neq 0$ ):

$$
\mathcal{X}_{1} \square \mathcal{X}_{2} \triangleq\left\{x \mid x=x_{1} / x_{2}, \text { where } x_{1} \in \mathcal{X}_{1}, x_{2} \in \mathcal{X}_{2}\right\}
$$

Operations with sets are associative and commutative similarly to operations with numbers. Thus, for $a, b, c, d, e, f \geq 0$ and $e, f>0$, if one computes $((a, b) \bullet(c, d)) \square$ $(e, f)$ one gets

$$
((a, b) \boxminus(c, d)) \square(e, f)=(a c, b d) \square(e, f)=(a c / f, b d / e)
$$

and we get the same result if we compute $(a, b) \bullet((c, d) \square(e, f))$ because

$$
(a, b) \boxminus((c, d) \square(e, f))=(a, b) \boxminus(c / f, d / e)=(a c / f, b d / e)
$$

In our next examples we always prefer to compute the divisions at the end since they often don't give exact values but approximations; and early approximations in calculations will grow in inacuracy.

\section{Imprecise Classic DSm fusion rule (imp-DSmC)}

The Imprecise Classic DSm fusion rule (imp-DSmC) which extends the Classic DSm fusion rule (DSmC) for combining imprecise (admissible) quantitative basic belief assignments is given for $k \geq 2$ sources by $m_{D S m C}^{I}(\emptyset)=0$ and $\forall A \neq \emptyset \in D^{\Theta}$,

$$
m_{D S m C}^{I}(A)=\sum_{\substack{X_{1}, X_{2}, \ldots, X_{k} \in D^{\Theta} \\\left(X_{1} \cap X_{2} \cap \ldots \cap X_{k}\right)=A}} \prod_{i=1, \ldots, k} m_{i}^{I}\left(X_{i}\right)
$$




\section{Imprecise Hybrid DSm fusion rule (imp-DSmH)}

Similarly, one can generalize (DSmH) from scalars to sets for the combination of $k \geq 2$ sources by $m_{D S m H}^{I}(\emptyset)=0$ and $\forall A \neq \emptyset \in D^{\Theta}$,

$$
m_{D S m H}^{I}(A) \triangleq \phi(A) \bullet\left[S_{1}^{I}(A) \boxplus S_{2}^{I}(A) \boxplus S_{3}^{I}(A)\right]
$$

with

$$
\begin{aligned}
& S_{1}^{I}(A) \triangleq \sum_{\substack{X_{1}, X_{2}, \ldots, X_{k} \in D^{\Theta} \\
\left(X_{1} \cap X_{2} \cap \ldots \cap X_{k}\right)=A}} \prod_{i=1, \ldots, k} m_{i}^{I}\left(X_{i}\right) \\
& S_{2}^{I}(A) \triangleq \sum_{\substack{X_{1}, X_{2}, \ldots, X_{k} \in \emptyset \\
[\mathcal{U}=A] \vee\left[\left(\mathcal{U} \in \boldsymbol{\emptyset} \wedge \wedge\left(A=I_{t}\right)\right]\right.}} \prod_{i=1, \ldots, k} m_{i}^{I}\left(X_{i}\right) \\
& S_{3}^{I}(A) \triangleq \sum_{\substack{X_{1}, X_{2}, \ldots, X_{k} \in D^{\Theta} \\
\left(c\left(X_{1} \cap X_{2} \cap \ldots \cap X_{k}\right)\right)=A \\
\left(X_{1} \cap X_{2} \cap \ldots \cap X_{k}\right) \in \emptyset}} \prod_{i=1, \ldots, k} m_{i}^{I}\left(X_{i}\right)
\end{aligned}
$$

These (imp-DSmC) and (imp-DSmH) fusion rules are just natural extensions of (DSmC) and $(\mathrm{DSmH})$ from scalar-valued to set-valued sources of information. It has been proved that (16) and (17) provide an admissible imprecise belief assignment (see the Theorem of Admissibility and its proof in Ch.6, p. 138, of [30]). In other words, DSm combinations of two admissible imprecise bba is also an admissible imprecise bba. As their precise counterparts, the imprecise DSm combination rules are quasi-associative, i.e. one stores in the computer's memory the conjunctive rule's result and, when new evidence comes in, this new evidence is combined with the conjunctive rule result. In this way the associativity is preserved.

\section{Imprecise PCR5 fusion rule (imp-PCR5)}

The (imp-PCR5) formula is a direct extension of (PCR5) formula using addition, multiplication and division operators on sets. It is given for the combination of $s \geq 2$ sources by $m_{P C R 5}^{I}(\emptyset)=0$ and $\forall X \in G \backslash\{\emptyset\}$ : 


$$
\begin{aligned}
& m_{P C R 5}^{I}(X)=\left[\sum_{\substack{X_{1}, X_{2}, \ldots, X_{s} \in G \\
\left(X_{1} \cap X_{2} \cap \ldots \cap X_{s}\right)=X}} \prod_{i=1, \ldots, s} m_{i}^{I}\left(X_{i}\right)\right] \\
& \boxplus\left[\sum_{\substack{2 \leq t \leq s \\
1 \leq r_{1}, \ldots, r_{t} \leq s}} \sum_{\substack{X_{j_{2}}, \ldots, X_{j_{t}} \in G \backslash\{X\} \\
\left\{j_{2}, \ldots, j_{t}\right\} \in \mathcal{P}^{t-1}(\{1, \ldots, n\})}}\left[\operatorname{Num}^{I}(X) \square \operatorname{Den}^{I}(X)\right]\right] \\
& 1 \leq r_{1}<r_{2}<\ldots<r_{t-1}<\left(r_{t}=s\right) \begin{array}{l}
\left\{j_{2}, \ldots, j_{t}\right\} \in P \\
c\left(X \cap X_{j_{2}} \cap \ldots \cap X_{j_{s}}\right)=\emptyset
\end{array} \\
& \left\{i_{1}, \ldots, i_{s}\right\} \in \mathcal{P}^{s}(\{1, \ldots, s\})
\end{aligned}
$$

where $\operatorname{Num}^{I}(X)$ and $\operatorname{Den}^{I}(X)$ are defined by

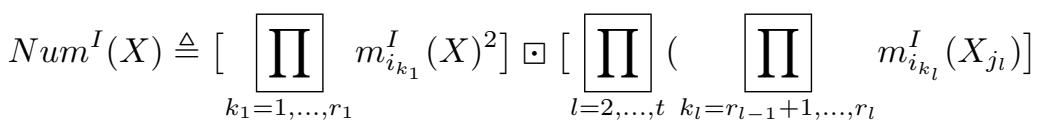

$$
\begin{aligned}
& \operatorname{Den}^{I}(X) \triangleq\left[\prod_{k_{1}=1, \ldots, r_{1}} m_{i_{k_{1}}}^{I}(X)\right] \boxplus\left[\sum_{l=2, \ldots, t}^{\left[\prod_{k_{l}=r_{l-1}+1, \ldots, r_{l}}\right.} m_{i_{k_{l}}}^{I}\left(X_{j_{l}}\right)\right]
\end{aligned}
$$

where all denominators-sets $\operatorname{Den}^{I}(X)$ involved in (21) are different from zero. If a denominator-set $\operatorname{Den}^{I}(X)$ is such that $\inf \left(\operatorname{Den}^{I}(X)\right)=0$, then the fraction is discarded. When $s=2$ (fusion of only two sources), the previous (imp-PCR5) formula reduces to its simple following fusion formula: $m_{P C R 5}^{I}(\emptyset)=0$ and $\forall X \in G \backslash\{\emptyset\}$

$$
\begin{aligned}
& m_{P C R 5}^{I}(X)=m_{12}^{I}(X)+ \\
& \sum_{\substack{Y \in G \backslash\{X\} \\
c(X \cap Y)=\emptyset}}\left[\left(m_{1}^{I}(X)^{2} m_{2}^{I}(Y)\right) \square\left(m_{1}^{I}(X)+m_{2}^{I}(Y)\right)\right] \boxplus \\
& {\left[\left(m_{2}^{I}(X)^{2} m_{1}^{I}(Y)\right) \square\left(m_{2}^{I}(X)+m_{1}^{I}(Y)\right)\right] }
\end{aligned}
$$

with

$$
m_{12}^{I}(X) \triangleq \sum_{\substack{X_{1}, X_{2} \in G \\ X_{1} \cap X_{2}=X}} m_{1}^{I}\left(X_{1}\right) \bowtie m_{2}^{I}\left(X_{2}\right)
$$




\begin{tabular}{|c|c|c|}
\hline$A \in D^{\Theta}$ & $m_{1}^{I}(A)$ & $m_{2}^{I}(A)$ \\
\hline$\theta_{1}$ & {$[0.1,0.2] \cup\{0.3\}$} & {$[0.4,0.5]$} \\
$\theta_{2}$ & $(0.4,0.6) \cup[0.7,0.8]$ & {$[0,0.4] \cup\{0.5,0.6\}$} \\
\hline
\end{tabular}

Table 1: Inputs of the fusion with imprecise bba

\section{Example for (imp-DSmC)}

Let's consider $\Theta=\left\{\theta_{1}, \theta_{2}\right\}$, two independent sources with the following imprecise admissible bba:

Using (imp-DSmC), i.e. the DSm classic rule for sets, one gets ${ }^{10}$

$$
\begin{aligned}
m_{D S m C}^{I}\left(\theta_{1}\right) & =([0.1,0.2] \cup\{0.3\}) \triangleright[0.4,0.5] \\
& =([0.1,0.2] \bullet[0.4,0.5]) \cup(\{0.3\} \bullet[0.4,0.5]) \\
& =[0.04,0.10] \cup[0.12,0.15] \\
m_{D S m C}^{I}\left(\theta_{2}\right)= & ((0.4,0.6) \cup[0.7,0.8]) \bullet([0,0.4] \cup\{0.5,0.6\}) \\
= & {[0,0.40] \cup[0.42,0.48] } \\
m_{D S m C}^{I}\left(\theta_{1} \cap \theta_{2}\right) & =[([0.1,0.2] \cup\{0.3\}) \triangleright([0,0.4] \cup\{0.5,0.6\})] \\
& =(0.16,0.58]
\end{aligned}
$$

Hence finally the fusion admissible result is given by:

\begin{tabular}{|c|c|}
\hline$A \in D^{\Theta}$ & $m_{D S m C}^{I}(A)=\left[m_{1}^{I} \oplus m_{2}^{I}\right](A)$ \\
\hline$\theta_{1}$ & {$[0.04,0.10] \cup[0.12,0.15]$} \\
$\theta_{2}$ & {$[0,0.40] \cup[0.42,0.48]$} \\
$\theta_{1} \cap \theta_{2}$ & $(0.16,0.58]$ \\
$\theta_{1} \cup \theta_{2}$ & 0 \\
\hline
\end{tabular}

Table 2: Fusion result with (imp-DSmC)

\section{Example for (imp-DSmH)}

If one finds out ${ }^{11}$ that $\theta_{1} \cap \theta_{2} \stackrel{\mathcal{M}}{\equiv} \emptyset$ (this is our hybrid model $\mathcal{M}$ one wants to deal with), then one uses the imprecise hybrid DSm rule (imp-DSmH) for sets (17) and

\footnotetext{
${ }^{10} \mathrm{~A}$ complete derivation of this reslut can be found in [30] pp. 139-140.

${ }^{11}$ We consider now a dynamic/temporal fusion problem.
} 
therefore the imprecise belief mass $m_{D S m C}^{I}\left(\theta_{1} \cap \theta_{2}\right)=(0.16,0.58]$ is then directly transferred onto $\theta_{1} \cup \theta_{2}$ and the others imprecise masses are not changed. Finally, the result obtained with (imp-DSmH) rule is given in Table 3.

\begin{tabular}{|c|c|}
\hline$A \in D^{\Theta}$ & $m_{D S m H}^{I}(A)=\left[m_{1}^{I} \oplus m_{2}^{I}\right](A)$ \\
\hline$\theta_{1}$ & {$[0.04,0.10] \cup[0.12,0.15]$} \\
$\theta_{2}$ & {$[0,0.40] \cup[0.42,0.48]$} \\
$\theta_{1} \cap \theta_{2} \stackrel{\mathcal{M}}{=}$ & 0 \\
$\theta_{1} \cup \theta_{2}$ & $(0.16,0.58]$ \\
\hline
\end{tabular}

Table 3: Fusion result with (imp-DSmH) for $\mathcal{M}(\Theta)$

We can easily check that for the source 1 , there exist the precise masses $\left(m_{1}\left(\theta_{1}\right)=\right.$ $0.3) \in([0.1,0.2] \cup\{0.3\})$ and $\left(m_{1}\left(\theta_{2}\right)=0.7\right) \in((0.4,0.6) \cup[0.7,0.8])$ such that $0.3+0.7=1$ and for the source 2 , there exist the precise masses $\left(m_{1}\left(\theta_{1}\right)=0.4\right) \in$ $([0.4,0.5])$ and $\left(m_{2}\left(\theta_{2}\right)=0.6\right) \in([0,0.4] \cup\{0.5,0.6\})$ such that $0.4+0.6=1$. Therefore both sources associated with $m_{1}^{I}($.$) and m_{2}^{I}($.$) are admissible imprecise sources$ of information. It can be easily checked that DSmC yields the paradoxical basic belief assignment $m_{D S m C}\left(\theta_{1}\right)=\left[m_{1} \oplus m_{2}\right]\left(\theta_{1}\right)=0.12, m_{D S m C}\left(\theta_{2}\right)=\left[m_{1} \oplus m_{2}\right]\left(\theta_{2}\right)=$ 0.42 and $m_{D S m C}\left(\theta_{1} \cap \theta_{2}\right)=\left[m_{1} \oplus m_{2}\right]\left(\theta_{1} \cap \theta_{2}\right)=0.46$. One sees from Table 2 that the admissibility is satisfied since there exists at least a bba (here $\left.m_{D S m C}().\right)$ with $\left(m_{D S m C}\left(\theta_{1}\right)=0.12\right) \in m_{D S m_{C}}^{I}\left(\theta_{1}\right),\left(m_{D S m C}\left(\theta_{2}\right)=0.42\right) \in m_{D S m C}^{I}\left(\theta_{2}\right)$ and $\left(m_{D S m C}\left(\theta_{1} \cap \theta_{2}\right)=0.46\right) \in m_{D S m C}^{I}\left(\theta_{1} \cap \theta_{2}\right.$ such that $0.12+0.42+0.46=1$.

Similarly if one finds out that $\theta_{1} \cap \theta_{2}=\emptyset$, then one uses DSmH and one gets: $m_{D S m H}\left(\theta_{1} \cap \theta_{2}\right)=0$ and $m_{D S m H}\left(\theta_{1} \cup \theta_{2}\right)=0.46$; the others remain unchanged. The admissibility still holds, because one can pick at least one number in each subset $m_{D S m H}^{I}($.$) such that the sum of these numbers is 1$. This approach can be also used in the similar manner to obtain imprecise pignistic probabilities from $m_{D S m H}^{I}($.$) for$ decision-making under quantitative uncertain, paradoxical and imprecise sources of information as well $[30,5]$.

\section{Examples for (imp-PCR5)}

\section{Example no 1:}

Let's consider $\Theta=\left\{\theta_{1}, \theta_{2}\right\}$, Shafer's model and two independent sources with the same imprecise admissible bba as those given in Table 1, i.e.

Working with sets, one gets for the conjunctive consensus

$$
m_{12}^{I}\left(\theta_{1}\right)=[0.04,0.10] \cup[0.12,0.15] \quad m_{12}^{I}\left(\theta_{2}\right)=[0,0.40] \cup[0.42,0.48]
$$




\begin{tabular}{|ll|}
\hline$m_{1}^{I}\left(\theta_{1}\right)=[0.1,0.2] \cup\{0.3\}$ & $m_{1}^{I}\left(\theta_{2}\right)=(0.4,0.6) \cup[0.7,0.8]$ \\
\hline$m_{2}^{I}\left(\theta_{1}\right)=[0.4,0.5]$ & $m_{2}^{I}\left(\theta_{2}\right)=[0,0.4] \cup\{0.5,0.6\}$ \\
\hline
\end{tabular}

while the conflicting imprecise mass is given by

$$
k_{12}^{I} \equiv m_{12}^{I}\left(\theta_{1} \cap \theta_{2}\right)=\left[m_{1}^{I}\left(\theta_{1}\right) \bowtie m_{2}^{I}\left(\theta_{2}\right)\right] \boxplus\left[m_{1}^{I}\left(\theta_{2}\right) \triangleright m_{2}^{I}\left(\theta_{1}\right)\right]=(0.16,0.58]
$$

Using the PCR5 rule for Proportional Conflict redistribution,

- one redistributes the partial imprecise conflicting mass $m_{1}^{I}\left(\theta_{1}\right) \bowtie m_{2}^{I}\left(\theta_{2}\right)$ to $\theta_{1}$ and $\theta_{2}$ proportionally to $m_{1}^{I}\left(\theta_{1}\right)$ and $m_{2}^{I}\left(\theta_{2}\right)$. Using the fraction bar symbol instead of $\square$ for convenience to denote the division operator on sets, one has

$$
\begin{aligned}
& \frac{x_{1}^{I}}{[0.1,0.2] \cup\{0.3\}=} \frac{y_{1}^{I}}{[0,0.4] \cup\{0.5,0.6\}} \\
&= \frac{([0.1,0.2] \cup\{0.3\}) \odot([0,0.4] \cup\{0.5,0.6\})}{([0.1,0.2] \cup\{0.3\}) \boxplus([0,0.4] \cup\{0.5,0.6\})} \\
&= {[[0,0.08] \cup[0.05,0.10] \cup[0.06,0.12]} \\
&\cup[0,0.12] \cup\{0.15,0.18\}] \\
& \\
& \square[[0.1,0.6] \cup[0.6,0.7] \cup[0.7,0.8] \\
&\cup[0.3,0.7] \cup\{0.8,0.9\}] \\
&=\frac{[0,0.12] \cup\{0.15,0.18\}}{[0.1,0.8] \cup\{0.9\}}
\end{aligned}
$$

whence

$$
\begin{aligned}
x_{1}^{I} & =\left[\frac{[0,0.12] \cup\{0.15,0.18\}}{[0.1,0.8] \cup\{0.9\}}\right] \bullet([0.1,0.2] \cup\{0.3\}) \\
& =\frac{[0,0.024] \cup[0.015,0.030] \cup[0.018,0.036] \cup[0,0.036] \cup\{0.045,0.048\}}{[0.1,0.8] \cup\{0.9\}} \\
& =\frac{[0,0.036] \cup\{0.045,0.048\}}{[0.1,0.8] \cup\{0.9\}} \\
& =\left[\frac{0}{0.8}, \frac{0.036}{0.1}\right] \cup\left[\frac{0}{0.9}, \frac{0.036}{0.9}\right] \cup\left[\frac{0.045}{0.8}, \frac{0.045}{0.1}\right] \cup\left[\frac{0.048}{0.8}, \frac{0.048}{0.1}\right] \\
& =[0,0.36] \cup[0,0.04] \cup[0.05625,0.45000] \cup[0.06,0.48]=[0,0.48]
\end{aligned}
$$




$$
\begin{aligned}
y_{1}^{I}= & {\left.\left[\frac{[0,0.12] \cup\{0.15,0.18\}}{[0.1,0.8] \cup\{0.9\}}\right] \bullet(0,0.4] \cup\{0.5,0.6\}\right) } \\
= & {[[0,0.048] \cup[0,0.060] \cup[0,0.072] \cup[0,0.6] \cup[0,0.072]} \\
& \cup\{0,075,0.090,0.090,0.108\}] \square[0.1,0.8] \cup\{0.9\} \\
= & \frac{[0,0.072] \cup\{0,075,0.090,0.108\}}{[0.1,0.8] \cup\{0.9\}} \\
= & {\left[\frac{0}{0.8}, \frac{0.072}{0.1}\right] \cup\left[\frac{0}{0.9}, \frac{0.072}{0.9}\right] \cup\left[\frac{0.075}{0.8}, \frac{0.075}{0.1}\right] } \\
& \cup\left[\frac{0.090}{0.8}, \frac{0.090}{0.1}\right] \cup\left[\frac{0.108}{0.8}, \frac{0.108}{0.1}\right] \cup\left\{\frac{0.075}{0.9}, \frac{0.090}{0.9}, \frac{0.108}{0.9}\right\} \\
= & {[0,0.72] \cup[0,0.08] \cup[0.09375,0.75] \cup[0.1125,0.9] \cup[0.135,1.08] } \\
& \cup\{0.083333,0.1,0.12\} \\
= & {[0,1.08] \approx[0,1] }
\end{aligned}
$$

- one redistributes the partial imprecise conflicting mass $m_{1}^{I}\left(\theta_{2}\right) \bullet m_{2}^{I}\left(\theta_{1}\right)$ to $\theta_{1}$ and $\theta_{2}$ proportionally to $m_{1}^{I}\left(\theta_{2}\right)$ and $m_{2}^{I}\left(\theta_{1}\right)$. One gets now the following proportionalization

$$
\begin{aligned}
\frac{x_{2}^{I}}{[0.4,0.5]} & =\frac{y_{2}^{I}}{(0.4,0.6) \cup[0.7,0.8]} \\
& =\frac{([0.4,0.5] \boxminus((0.4,0.6) \cup[0.7,0.8])}{([0.4,0.5] \boxplus((0.4,0.6) \cup[0.7,0.8])} \\
& =\frac{(0.16,0.30) \cup[0.28,0.40]}{(0.8,1.1) \cup[1.1,1.3]}=\frac{(0.16,0.40]}{(0.8,1.3]}
\end{aligned}
$$

whence

$$
\begin{aligned}
x_{2}^{I} & =\frac{(0.16,0.40]}{(0.8,1.3]} \bullet[0.4,0.5] \\
& =\frac{(0.064,0.200]}{(0.8,1.3]} \\
& =\left(\frac{0.064}{1.3}, \frac{0.200}{0.8}\right)=(0.049231,0.250000)
\end{aligned}
$$




$$
\begin{aligned}
y_{2}^{I} & =\frac{(0.16,0.40]}{(0.8,1.3]} \odot(0.4,0.6) \cup[0.7,0.8] \\
& =\frac{(0.064,0.240) \cup(0.112,0.320]}{(0.8,1.3]} \\
& =\frac{(0.064,0.320]}{(0.8,1.3]}=\left(\frac{0.064}{1.3}, \frac{0.320}{0.8}\right) \\
& =(0.049231,0.400000)
\end{aligned}
$$

Hence, one finally gets with imprecise PCR5,

$$
\begin{aligned}
m_{P C R 5}^{I}\left(\theta_{1}\right) & =m_{12}^{I}\left(\theta_{1}\right) \boxplus x_{1}^{I} \boxplus x_{2}^{I} \\
& =([0.04,0.10] \cup[0.12,0.15]) \boxplus[0,0.48] \boxplus(0.049231,0.250000) \\
& =([0.04,0.10] \cup[0.12,0.15]) \boxplus(0.049231,0.73) \\
& =(0.089231,0.83) \cup(0.169231,0.88) \\
& =(0.089231,0.88) \\
m_{P C R 5}^{I}\left(\theta_{2}\right) & =m_{12}^{I}\left(\theta_{2}\right) \boxplus y_{1}^{I} \boxplus y_{2}^{I} \\
& =([0,0.40] \cup[0.42,0.48]) \boxplus[0,1] \boxplus(0.049231,0.400000) \approx[0,1] \\
m_{P C R 5}^{I}\left(\theta_{1} \cap \theta_{2}\right) & =0
\end{aligned}
$$

Example no 2:

Let's consider a more simple example with $\Theta=\left\{\theta_{1}, \theta_{2}\right\}$, Shafer's model and two independent sources with the following imprecise admissible bba

$$
\begin{array}{|ll|}
\hline m_{1}^{I}\left(\theta_{1}\right)=(0.2,0.3) & m_{1}^{I}\left(\theta_{2}\right)=[0.6,0.8] \\
\hline m_{2}^{I}\left(\theta_{1}\right)=[0.4,0.7) & m_{2}^{I}\left(\theta_{2}\right)=(0.5,0.6] \\
\hline
\end{array}
$$

Working with sets, one gets for the conjunctive consensus

$$
m_{12}^{I}\left(\theta_{1}\right)=(0.08,0.21) \quad m_{12}^{I}\left(\theta_{2}\right)=(0.30,0.48)
$$

The total (imprecise) conflict between the two imprecise quantitative sources is given by

$$
\begin{aligned}
k_{12}^{I} \equiv m_{12}^{I}\left(\theta_{1} \cap \theta_{2}\right) & =\left[m_{1}^{I}\left(\theta_{1}\right) \bowtie m_{2}^{I}\left(\theta_{2}\right)\right] \boxplus\left[m_{1}^{I}\left(\theta_{2}\right) \bowtie m_{2}^{I}\left(\theta_{1}\right)\right] \\
& =((0.2,0.3) \triangleright(0.5,0.6]) \boxplus([0.4,0.7] \oplus[0.6,0.8]) \\
& =(0.10,0.18) \boxplus[0.24,0.56)=(0.34,0.74)
\end{aligned}
$$


Using the PCR5 rule for Proportional Conflict redistribution of partial (imprecise) conflict $m_{1}^{I}\left(\theta_{1}\right) \bullet m_{2}^{I}\left(\theta_{2}\right)$, one has

$$
\frac{x_{1}^{I}}{(0.2,0.3)}=\frac{y_{1}^{I}}{(0.5,0.6]}=\frac{(0.2,0.3) \oplus(0.5,0.6]}{(0.2,0.3) \boxplus(0.5,0.6]}=\frac{(0.10,0.18)}{(0.7,0.9)}
$$

whence

$$
\begin{aligned}
x_{1}^{I} & =\frac{(0.10,0.18)}{(0.7,0.9)} \odot(0.2,0.3) \\
& =\frac{(0.02,0.054)}{(0.7,0.9)} \\
& =\left(\frac{0.02}{0.9}, \frac{0.054}{0.7}\right) \\
& =(0.022222,0.077143) \\
y_{1}^{I} & =\frac{(0.10,0.18)}{(0.7,0.9)} \bullet(0.5,0.6] \\
& =\frac{(0.050,0.108)}{(0.7,0.9)} \\
& =\left(\frac{0.050}{0.9}, \frac{0.108}{0.7}\right) \\
& =(0.055556,0.154286)
\end{aligned}
$$

Using the PCR5 rule for Proportional Conflict redistribution of partial (imprecise) conflict $m_{1}^{I}\left(\theta_{2}\right) \bullet m_{2}^{I}\left(\theta_{1}\right)$, one has

$$
\frac{x_{2}^{I}}{[0.4,0.7)}=\frac{y_{2}^{I}}{[0.6,0.8]}=\frac{[0.4,0.7) \oplus[0.6,0.8]}{[0.4,0.7) \boxplus[0.6,0.8]}=\frac{[0.24,0.56)}{[1,1.5)}
$$

whence

$$
\begin{aligned}
& x_{2}^{I}=\frac{[0.24,0.56)}{[1,1.5)} \oslash[0.4,0.7)=\frac{[0.096,0.392)}{[1,1.5)}=\left(\frac{0.096}{1.5}, \frac{0.392}{1}\right)=(0.064,0.392) \\
& y_{2}^{I}=\frac{[0.24,0.56)}{[1,1.5)} \oslash[0.6,0.8]=\frac{[0.144,0.448)}{[1,1.5)}=\left(\frac{0.144}{1.5}, \frac{0.448}{1}\right)=(0.096,0.448)
\end{aligned}
$$

Hence, one finally gets with imprecise PCR5, 


$$
\begin{aligned}
m_{P C R 5}^{I}\left(\theta_{1}\right) & =m_{12}^{I}\left(\theta_{1}\right) \boxplus x_{1}^{I} \boxplus x_{2}^{I} \\
& =(0.08,0.21) \boxplus(0.022222,0.077143) \boxplus(0.064,0.392) \\
& =(0.166222,0.679143) \\
m_{P C R 5}^{I}\left(\theta_{2}\right) & =m_{12}^{I}\left(\theta_{2}\right) \boxplus y_{1}^{I} \boxplus y_{2}^{I} \\
& =(0.30,0.48) \boxplus(0.055556,0.154286) \boxplus(0.096,0.448) \\
& =(0.451556,1.08229) \approx(0.451556,1] \\
m_{P C R 5}^{I}\left(\theta_{1} \cap \theta_{2}\right) & =0
\end{aligned}
$$

\section{Fusion of qualitative beliefs}

Different qualitative methods for reasoning under uncertainty have been developed mainly in Artificial Intelligence since the last decades. They attract more and more people of Information Fusion community, specially those working in the development of modern multi-source ${ }^{12}$ systems for defense. George Polya was the first mathematician to attempt a formal characterization of qualitative human reasoning in 1954 [27], then followed by Lofti Zadeh's works [44]-[51]. The interest of qualitative reasoning methods is to help in decision-making for situations in which the precise numerical methods are not appropriate (whenever the information/input are not directly expressed in numbers). Several formalisms for qualitative reasoning have been proposed as extensions on the frames of probability, possibility and/or evidence theories $[1,11,4,40,17,48,51,43]$. The limitations of numerical techniques are discussed in [23]. Our purpose here is not to browse and to write a survey on all techniques dealing with qualitative information, but only to mention briefly the main attempts for solving the combination problem. A good presentation of these techniques can be found in Parsons' milestone book [25]. Among all available techniques, one must however give credit to Wellman's works [39] who proposed a general characterization of "qualitative probability" to relax precision in representation and reasoning within the probabilistic framework. His "qualitative" Probabilistic Networks (QPN) based on a Qualitative Probability Language (QPL) defined by a set of numerical underlying probability distributions belongs actually to the family of imprecise probability [38] and probability bounds analysis (PBA) methods [12] and cannot be considered truly as a qualitative approach since it deals with quantitative (imprecise) probability distributions. Based on Dempster-Shafer Theory, Wong and Lingras [41] proposed a method for generating a (numerical) basic belief function from preference relations between each pair of propositions specified qualitatively. Their method doesn't provide however a unique solution and doesn't check the consistency of qualitative preference relations and cannot be truly considered as a full qualitative method. Bryson et al. [3, 20] proposed

\footnotetext{
${ }^{12}$ Where both computers, sensors and human experts are involved in the loop.
} 
a Qualitative Discriminant Procedure (QDP) that involves qualitative scoring, imprecise pairwise comparisons between pairs of propositions and an optimization algorithm to generate consistent imprecise quantitative belief function to combine. In [21, 22], Parsons proposed for the first time (upon the knowledge of the authors) a qualitative Dempster-Shafer Theory (QET), by using techniques from qualitative reasoning [1]. Based on operation tables, he introduced a very simple arithmetic for qualitative addition + and multiplication $\times$ operators. Because of impossibility of qualitative normalization, Parsons used the un-normalized version of Dempster's rule by committing a qualitative mass to the empty set following the open-world approach of Smets [35]. This approach cannot deal however with truly closed-world problems because there is unfortunately no issue to transfer the conflicting qualitative mass or to normalize the qualitative belief assignments in the spirit of DST. Since 1998, Parsons started to develop Qualitative Probabilistic Reasoner (QPR) [24, 26]. Since middle of nineties, Lofti Zadeh has proposed a new paradigm of computing with words (CW) [48]-[51] to combine qualitative/vague information expressed in natural language. $\mathrm{CW}$ is done essentially in three major steps: 1) a translation of qualitative information into fuzzy membership functions, 2) a fuzzy combination of fuzzy membership functions; 3) a retranslation of fuzzy (quantitative) result into natural language. All these steps cannot be uniquely accomplished since they depend on the fuzzy operators chosen. A possible issue for the third step is proposed in [43].

In this section we propose a simple arithmetic of linguistic labels which allows a direct extension of classical (quantitative) combination rules proposed in the DSmT framework into their qualitative counterpart. Qualitative beliefs assignments are well adapted for manipulated information expressed in natural language and usually reported by human expert or AI-based expert systems. In other words, we propose here a new method for computing directly with words $(\mathrm{CW})$ and combining directly qualitative information Computing with words, more precisely computing with linguistic labels, is usually more vague, less precise than computing with numbers, but it is expected to offer a better robustness and flexibility for combining uncertain and conflicting human reports than computing with numbers because in most of cases human experts are less efficient to provide (and to justify) precise quantitative beliefs than qualitative beliefs. Before extending the quantitative DSmT-based combination rules to their qualitative counterparts, it will be necessary to define few but new important operators on linguistic labels and what is a qualitative belief assignment. Then we will show though simple examples how the combination of qualitative beliefs can be obtained in the DSmT framework.

\subsection{Qualitative Operators}

Let's define a finite set of linguistic labels $\tilde{L}=\left\{L_{1}, L_{2}, \ldots, L_{m}\right\}$ where $m \geq 2$ is an integer. $\tilde{L}$ is endowed with a total order relationship $\prec$, so that $L_{1} \prec L_{2} \prec \ldots \prec L_{m}$. 
To work on a close linguistic set under linguistic addition and multiplication operators, we extends $\tilde{L}$ with two extreme values $L_{0}$ and $L_{m+1}$ where $L_{0}$ corresponds to the minimal qualitative value and $L_{m+1}$ corresponds to the maximal qualitative value, in such a way that

$$
L_{0} \prec L_{1} \prec L_{2} \prec \ldots \prec L_{m} \prec L_{m+1}
$$

where $\prec$ means inferior to, or less (in quality) than, or smaller (in quality) than, etc. hence a relation of order from a qualitative point of view. But if we make a correspondence between qualitative labels and quantitative values on the scale $[0,1]$, then $L_{\min }=L_{0}$ would correspond to the numerical value 0 , while $L_{\max }=L_{m+1}$ would correspond to the numerical value 1 , and each $L_{i}$ would belong to $[0,1]$, i. e.

$$
L_{\min }=L_{0}<L_{1}<L_{2}<\ldots<L_{m}<L_{m+1}=L_{\max }
$$

From now on, we work on extended ordered set $L$ of qualitative values

$$
L=\left\{L_{0}, \tilde{L}, L_{m+1}\right\}=\left\{L_{0}, L_{1}, L_{2}, \ldots, L_{m}, L_{m+1}\right\}
$$

The qualitative addition and multiplication operators are respectively defined in the following way:

- Addition :

$$
L_{i}+L_{j}= \begin{cases}L_{i+j}, & \text { if } i+j \leq m+1 \\ L_{m+1}, & \text { if } i+j>m+1\end{cases}
$$

- Multiplication :

$$
L_{i} \times L_{j}=L_{\min \{i, j\}}
$$

These two operators are well-defined, commutative, associative, and unitary. Addition of labels is a unitary operation since $L_{0}=L_{\min }$ is the unitary element, i.e. $L_{i}+L_{0}=L_{0}+L_{i}=L_{i+0}=L_{i}$ for all $0 \leq i \leq m+1$. Multiplication of labels is also a unitary operation since $L_{m+1}=L_{\max }$ is the unitary element, i.e. $L_{i} \times L_{m+1}=L_{m+1} \times L_{i}=L_{\min \{i, m+1\}}=L_{i}$ for $0 \leq i \leq m+1 . L_{0}$ is the unit element for addition, while $L_{m+1}$ is the unit element for multiplication. $L$ is closed under + and $\times$. The mathematical structure formed by $(L,+, \times)$ is a commutative bisemigroup with different unitary elements for each operation. We recall that a bisemigroup is a set $S$ endowed with two associative binary operations such that $S$ is closed under both operations.

If $L$ is not an exhaustive set of qualitative labels, then other labels may exist in between the initial ones, so we can work with labels and numbers - since a refinement of $L$ is possible. When mapping from $L$ to crisp numbers or intervals, $L_{0}=0$ and $L_{m+1}=1$, while $0<L_{i}<1$, for all $i$, as crisp numbers, or $L_{i}$ included in $[0,1]$ as intervals/subsets. 
For example, $L_{1}, L_{2}, L_{3}$ and $L_{4}$ may represent the following qualitative values: $L_{1} \triangleq$ very poor, $L_{2} \triangleq$ poor, $L_{3} \triangleq \operatorname{good}$ and $L_{4} \triangleq$ very good where $\triangleq$ symbol means "by definition".

We think it is better to define the multiplication $\times$ of $L_{i} \times L_{j}$ by $L_{\min \{i, j\}}$ because multiplying two numbers $a$ and $b$ in $[0,1]$ one gets a result which is less than each of them, the product is not bigger than both of them as Bolanos et al. did in [2] by approximating $L_{i} \times L_{j}=L_{i+j}>\max \left\{L_{i}, L_{j}\right\}$. While for the addition it is the opposite: adding two numbers in the interval $[0,1]$ the sum should be bigger than both of them, not smaller as in [2] case where $L_{i}+L_{j}=\min \left\{L_{i}, L_{j}\right\}<\max \left\{L_{i}, L_{j}\right\}$.

\subsection{Qualitative Belief Assignment}

We define a qualitative belief assignment (qba), and we call it qualitative belief mass or $q$-mass for short, a mapping function

$$
q m(.): G \mapsto L
$$

where $G$ corresponds the space of propositions generated with $\cap$ and $\cup$ operators and elements of $\Theta$ taking into account the integrity constraints of the model. For example if Shafer's model is chosen for $\Theta$, then $G$ is nothing but the classical power set $2^{\Theta}$ [29], whereas if free DSm model is adopted $G$ will correspond to Dedekind's lattice (hyper-power set) $D^{\Theta}$ [30]. Note that in this qualitative framework, there is no way to define normalized $q m($.$) , but qualitative quasi-normalization is still possible as seen$ further. Using the qualitative operations defined previously we can easily extend the combination rules from quantitative to qualitative. In the sequel we will consider $s \geq 2$ qualitative belief assignments $q m_{1}(),. \ldots, q m_{s}($.$) defined over the same space G$ and provided by $s$ independent sources $S_{1}, \ldots, S_{s}$ of evidence.

Important note: The addition and multiplication operators used in all qualitative fusion formulas in next sections correspond to qualitative addition and qualitative multiplication operators defined in (25) and (26) and must not be confused with classical addition and multiplication operators for numbers.

\subsection{Qualitative Conjunctive Rule (qCR)}

The qualitative Conjunctive Rule (qCR) of $s \geq 2$ sources is defined similarly to the quantitative conjunctive consensus rule, i.e.

$$
q m_{q C R}(X)=\sum_{\substack{X_{1}, \ldots, X_{s} \in G \\ X_{1} \cap \ldots \cap X_{s}=X}} \prod_{i=1}^{s} q m_{i}\left(X_{i}\right)
$$


The total qualitative conflicting mass is given by

$$
K_{1 \ldots s}=\sum_{\substack{X_{1}, \ldots, X_{s} \in G \\ X_{1} \cap \ldots \cap X_{s}=\emptyset}} \prod_{i=1}^{s} q m_{i}\left(X_{i}\right)
$$

\subsection{Qualitative DSm Classic rule (q-DSmC)}

The qualitative DSm Classic rule (qDSmC) for $s \geq 2$ is defined similarly to DSm Classic fusion rule (DSmC) as follows : $q m_{q D S m C}(\emptyset)=L_{0}$ and for all $X \in D^{\Theta} \backslash\{\emptyset\}$,

$$
q m_{q D S m C}(X)=\sum_{\substack{X_{1}, \ldots, X_{s} \in D^{\Theta} \\ X_{1} \cap \ldots \cap X_{s}=X}} \prod_{i=1}^{s} q m_{i}\left(X_{i}\right)
$$

\subsection{Qualitative DSm Hybrid rule (q-DSmH)}

The qualitative DSm Hybrid rule (qDSmH) is defined similarly to quantitative DSm hybrid rule [30] as follows: $q m_{q D S m H}(\emptyset)=L_{0}$ and for all $X \in G \backslash\{\emptyset\}$

$$
q m_{q D S m H}(X) \triangleq \phi(X) \cdot\left[q S_{1}(X)+q S_{2}(X)+q S_{3}(X)\right]
$$

where $\phi(X)$ is the characteristic non-emptiness function of a set $X$, i.e. $\phi(X)=L_{m+1}$ if $X \notin \emptyset$ and $\phi(X)=L_{0}$ otherwise, where $\emptyset \triangleq\left\{\emptyset_{\mathcal{M}}, \emptyset\right\}$. $\emptyset_{\mathcal{M}}$ is the set of all elements of $D^{\Theta}$ which have been forced to be empty through the constraints of the model $\mathcal{M}$ and $\emptyset$ is the classical/universal empty set. $q S_{1}(X) \equiv q m_{q D S m C}(X), q S_{2}(X), q S_{3}(X)$ are defined by

$$
\begin{gathered}
q S_{1}(X) \triangleq \sum_{\substack{X_{1}, X_{2}, \ldots, X_{s} \in D^{\Theta} \\
\left(X_{1} \cap X_{2} \cap \ldots \cap X_{s}\right)=X}} \prod_{i=1}^{s} q m_{i}\left(X_{i}\right) \\
q S_{2}(X) \triangleq \sum_{\substack{\left.X_{1}, X_{2}, \ldots, X_{s} \in \emptyset \\
\mathcal{U}=X\right] \vee\left[(\mathcal{U} \in \emptyset) \wedge\left(X=I_{t}\right)\right]}} \prod_{i=1}^{s} q m_{i}\left(X_{i}\right) \\
q S_{3}(X) \triangleq \sum_{\substack{X_{1}, X_{2}, \ldots, X_{k} \in D^{\Theta} \\
u\left(c\left(X_{1} \cap X_{2} \cap \ldots \cap X_{s}\right)\right)=X \\
\left(X_{1} \cap X_{2} \cap \ldots \cap X_{s}\right) \in \emptyset}}^{s} \prod_{i=1}^{s} q m_{i}\left(X_{i}\right)
\end{gathered}
$$

with $\mathcal{U} \triangleq u\left(X_{1}\right) \cup \ldots \cup u\left(X_{s}\right)$ where $u(X)$ is the union of all $\theta_{i}$ that compose $X$, $I_{t} \triangleq \theta_{1} \cup \ldots \cup \theta_{n}$ is the total ignorance, and $c(X)$ is the canonical form of $X$, i.e. its simplest form (for example if $X=(A \cap B) \cap(A \cup B \cup C), c(X)=A \cap B)$. $q S_{1}(X)$ 
is nothing but the qDSmC rule for $s$ independent sources based on $\mathcal{M}^{f}(\Theta) ; q S_{2}(X)$ is the qualitative mass of all relatively and absolutely empty sets which is transferred to the total or relative ignorances associated with non existential constraints (if any, like in some dynamic problems); $q S_{3}(X)$ transfers the sum of relatively empty sets directly onto the canonical disjunctive form of non-empty sets. qDSmH generalizes qDSmC works for any models (free DSm model, Shafer's model or any hybrid models) when manipulating qualitative belief assignments.

\subsection{Qualitative PCR5 rule (q-PCR5)}

In classical/quantitative DSmT framework, the Proportional Conflict Redistribution rule no. 5 (PCR5) has been proven to provide very good and coherent results for combining (quantitative) belief masses [32, 19,9]. When dealing with qualitative beliefs and using Dempster-Shafer Theory (DST), we unfortunately can not normalize, since it is not possible to divide linguistic labels by linguistic labels. Previous authors have used the un-normalized Dempster's rule, which actually is equivalent to the Conjunctive Rule in Shafer's model and respectively to DSm conjunctive rule in hybrid and free DSm models. Following the idea of (quantitative) PCR5 fusion rule (9), we can however use a rough approximation for a qualitative version of PCR5 (denoted qPCR5) as it will be presented in next example, but we did not succeed so far to get a general formula for qualitative PCR5 fusion rule (q-PCR5) because the division of labels could not be defined.

\subsection{Example}

Let's consider the following set of ordered linguistic labels $L=\left\{L_{0}, L_{1}, L_{2}, L_{3}, L_{4}, L_{5}\right\}$ (for example, $L_{1}, L_{2}, L_{3}$ and $L_{4}$ may represent the values: $L_{1} \triangleq$ very poor, $L_{2} \triangleq$ poor, $L_{3} \triangleq \operatorname{good}$ and $L_{4} \triangleq$ very good, where $\triangleq$ symbol means by definition), then addition and multiplication tables are

\begin{tabular}{|c|cccccc|}
\hline+ & $L_{0}$ & $L_{1}$ & $L_{2}$ & $L_{3}$ & $L_{4}$ & $L_{5}$ \\
\hline$L_{0}$ & $L_{0}$ & $L_{1}$ & $L_{2}$ & $L_{3}$ & $L_{4}$ & $L_{5}$ \\
$L_{1}$ & $L_{1}$ & $L_{2}$ & $L_{3}$ & $L_{4}$ & $L_{5}$ & $L_{5}$ \\
$L_{2}$ & $L_{2}$ & $L_{3}$ & $L_{4}$ & $L_{5}$ & $L_{5}$ & $L_{5}$ \\
$L_{3}$ & $L_{3}$ & $L_{4}$ & $L_{5}$ & $L_{5}$ & $L_{5}$ & $L_{5}$ \\
$L_{4}$ & $L_{4}$ & $L_{5}$ & $L_{5}$ & $L_{5}$ & $L_{5}$ & $L_{5}$ \\
$L_{5}$ & $L_{5}$ & $L_{5}$ & $L_{5}$ & $L_{5}$ & $L_{5}$ & $L_{5}$ \\
\hline
\end{tabular}

Table 4: Addition table

Let's consider now a simple two-source case with a 2D frame $\Theta=\left\{\theta_{1}, \theta_{2}\right\}$, Shafer's model for $\Theta$, and qba's expressed as follows:

$$
q m_{1}\left(\theta_{1}\right)=L_{1}, \quad q m_{1}\left(\theta_{2}\right)=L_{3}, \quad q m_{1}\left(\theta_{1} \cup \theta_{2}\right)=L_{1}
$$




\begin{tabular}{|c|llllll|}
\hline$\times$ & $L_{0}$ & $L_{1}$ & $L_{2}$ & $L_{3}$ & $L_{4}$ & $L_{5}$ \\
\hline$L_{0}$ & $L_{0}$ & $L_{0}$ & $L_{0}$ & $L_{0}$ & $L_{0}$ & $L_{0}$ \\
$L_{1}$ & $L_{0}$ & $L_{1}$ & $L_{1}$ & $L_{1}$ & $L_{1}$ & $L_{1}$ \\
$L_{2}$ & $L_{0}$ & $L_{1}$ & $L_{2}$ & $L_{2}$ & $L_{2}$ & $L_{2}$ \\
$L_{3}$ & $L_{0}$ & $L_{1}$ & $L_{2}$ & $L_{3}$ & $L_{3}$ & $L_{3}$ \\
$L_{4}$ & $L_{0}$ & $L_{1}$ & $L_{2}$ & $L_{3}$ & $L_{4}$ & $L_{4}$ \\
$L_{5}$ & $L_{0}$ & $L_{1}$ & $L_{2}$ & $L_{3}$ & $L_{4}$ & $L_{5}$ \\
\hline
\end{tabular}

Table 5: Multiplication table

$$
q m_{2}\left(\theta_{1}\right)=L_{2}, \quad q m_{2}\left(\theta_{2}\right)=L_{1}, \quad q m_{2}\left(\theta_{1} \cup \theta_{2}\right)=L_{2}
$$

- Fusion with (qCR): According to qCR combination rule (27), one gets the result in Table 6 , since

$$
\begin{aligned}
& q m_{q C R}\left(\theta_{1}\right)=q m_{1}\left(\theta_{1}\right) q m_{2}\left(\theta_{1}\right)+q m_{1}\left(\theta_{1}\right) q m_{2}\left(\theta_{1} \cup \theta_{2}\right) \\
& +q m_{2}\left(\theta_{1}\right) q m_{1}\left(\theta_{1} \cup \theta_{2}\right) \\
& =\left(L_{1} \times L_{2}\right)+\left(L_{1} \times L_{2}\right)+\left(L_{2} \times L_{1}\right) \\
& =L_{1}+L_{1}+L_{1}=L_{1+1+1}=L_{3} \\
& q m_{q C R}\left(\theta_{2}\right)=q m_{1}\left(\theta_{2}\right) q m_{2}\left(\theta_{2}\right)+q m_{1}\left(\theta_{2}\right) q m_{2}\left(\theta_{1} \cup \theta_{2}\right) \\
& +q m_{2}\left(\theta_{2}\right) q m_{1}\left(\theta_{1} \cup \theta_{2}\right) \\
& =\left(L_{3} \times L_{1}\right)+\left(L_{3} \times L_{2}\right)+\left(L_{1} \times L_{1}\right) \\
& =L_{1}+L_{2}+L_{1}=L_{1+2+1}=L_{4} \\
& q m_{q C R}\left(\theta_{1} \cup \theta_{2}\right)=q m_{1}\left(\theta_{1} \cup \theta_{2}\right) q m_{2}\left(\theta_{1} \cup \theta_{2}\right)=L_{1} \times L_{2}=L_{1} \\
& q m_{q C R}(\emptyset) \triangleq K_{12}=q m_{1}\left(\theta_{1}\right) q m_{2}\left(\theta_{2}\right)+q m_{1}\left(\theta_{2}\right) q m_{2}\left(\theta_{1}\right) \\
& =\left(L_{1} \times L_{1}\right)+\left(L_{2} \times L_{3}\right)=L_{1}+L_{2}=L_{3}
\end{aligned}
$$

In summary, one gets

- Fusion with (qDSmC): If we accepts the free-DSm model instead Shafer's model, according to qDSmC combination rule (28), one gets the result in Table 7 , 


\begin{tabular}{|l|ccccc|}
\hline & $\theta_{1}$ & $\theta_{2}$ & $\theta_{1} \cup \theta_{2}$ & $\emptyset$ & $\theta_{1} \cap \theta_{2}$ \\
\hline$q m_{1}()$. & $L_{1}$ & $L_{3}$ & $L_{1}$ & & \\
$q m_{2}()$. & $L_{2}$ & $L_{1}$ & $L_{2}$ & & \\
\hline$q m_{q C R}()$. & $L_{3}$ & $L_{4}$ & $L_{1}$ & $L_{3}$ & $L_{0}$ \\
\hline
\end{tabular}

Table 6: Fusion with qCR

\begin{tabular}{|l|ccccc|}
\hline & $\theta_{1}$ & $\theta_{2}$ & $\theta_{1} \cup \theta_{2}$ & $\emptyset$ & $\theta_{1} \cap \theta_{2}$ \\
\hline$q m_{1}()$. & $L_{1}$ & $L_{3}$ & $L_{1}$ & & \\
$q m_{2}()$. & $L_{2}$ & $L_{1}$ & $L_{2}$ & & \\
\hline$q m_{q D S m C}()$. & $L_{3}$ & $L_{4}$ & $L_{1}$ & $L_{0}$ & $L_{3}$ \\
\hline
\end{tabular}

Table 7: Fusion with qDSmC

- Fusion with (qDSmH): Working with Shafer's model for $\Theta$, according to qDSmH combination rule (29), one gets the result in Table 8.

since $q m_{q D S m H}\left(\theta_{1} \cup \theta_{2}\right)=L_{1}+L_{3}=L_{4}$.

- Fusion with (qPCR5): Following PCR5 method, we propose to transfer the qualitative partial masses

a) $q m_{1}\left(\theta_{1}\right) q m_{2}\left(\theta_{2}\right)=L_{1} \times L_{1}=L_{1}$ to $\theta_{1}$ and $\theta_{2}$ in equal parts (i.e. proportionally to $L_{1}$ and $L_{1}$ respectively, but $L_{1}=L_{1}$ ); hence $\frac{1}{2} L_{1}$ should go to each of them.

b) $q m_{2}\left(\theta_{1}\right) q m_{1}\left(\theta_{2}\right)=L_{2} \times L_{3}=L_{2}$ to $\theta_{1}$ and $\theta_{2}$ proportionally to $L_{2}$ and $L_{3}$ respectively; but since we are not able to do an exact proportionalization of labels, we approximate through transferring $\frac{1}{3} L_{2}$ to $\theta_{1}$ and $\frac{2}{3} L_{2}$ to $\theta_{2}$.

The transfer $1 / 3 L_{2}$ to $\theta_{1}$ and $2 / 3 L_{2}$ to $\theta_{2}$ is not arbitrary, but it is an approximation since the transfer was done proportionally to $L_{2}$ and $L_{3}$, and $L_{2}$ is smaller than $L_{3}$; we mention that it is not possible to do an exact transferring. Nobody in the literature has done so far normalization of labels, and we tried to do a quasi-normalization [i.e. an approximation].

Summing a) and b) we get: $\frac{1}{2} L_{1}+\frac{1}{3} L_{2} \approx L_{1}$, which represents the partial conflicting qualitative mass transferred to $\theta_{1}$, and $\frac{1}{2} L_{1}+\frac{2}{3} L_{2} \approx L_{2}$, which represents the partial conflicting qualitative mass transferred to $\theta_{2}$. Here we have mixed qualitative and quantitative information.

Hence we will finally get:

Fore the reason that we can not do a normalization (neither previous authors on qualitative fusion rules did), we propose for the first time the possibility of 


\begin{tabular}{|l|ccccc|}
\hline & $\theta_{1}$ & $\theta_{2}$ & $\theta_{1} \cup \theta_{2}$ & $\emptyset$ & $\theta_{1} \cap \theta_{2}$ \\
\hline$q m_{1}()$. & $L_{1}$ & $L_{3}$ & $L_{1}$ & & \\
$q m_{2}()$. & $L_{2}$ & $L_{1}$ & $L_{2}$ & & \\
\hline$q m_{q D S m H}()$. & $L_{3}$ & $L_{4}$ & $L_{4}$ & $L_{0}$ & $L_{0}$ \\
\hline
\end{tabular}

Table 8: Fusion with qDSmC

\begin{tabular}{|l|ccccc|}
\hline & $\theta_{1}$ & $\theta_{2}$ & $\theta_{1} \cup \theta_{2}$ & $\emptyset$ & $\theta_{1} \cap \theta_{2}$ \\
\hline$q m_{1}()$. & $L_{1}$ & $L_{3}$ & $L_{1}$ & & \\
$q m_{2}()$. & $L_{2}$ & $L_{1}$ & $L_{2}$ & & \\
\hline$q m_{q P C R 5}()$. & $L_{4}$ & $L_{5}$ & $L_{1}$ & $L_{0}$ & $L_{0}$ \\
\hline
\end{tabular}

Table 9: Fusion with qPCR5

quasi-normalization (which is an approximation of the normalization), i.e. instead of dividing each qualitative mass by a coefficient of normalization, we subtract from each qualitative mass a qualitative coefficient (label) of quasinormalization in order to adjust the sum of masses.

Subtraction on $L$ is defined in a similar way to the addition:

$$
L_{i}-L_{j}= \begin{cases}L_{i-j}, & \text { if } i \geq j \\ L_{0}, & \text { if } i<j\end{cases}
$$

$L$ is closed under subtraction as well.

The subtraction can be used for quasi-normalization only, i. e. moving the final label result 1-2 steps/labels up or down. It is not used together with addition or multiplication.

The increment in the sum of fusioned qualitative masses is due to the fact that multiplication on $L$ is approximated by a larger number, because multiplying any two numbers $a, b$ in the interval $[0,1]$, the product is less than each of them, or we have approximated the product $a \times b=\min \{a, b\}$.

Using the quasi-normalization (subtracting $L_{1}$ ), one gets with qDSmH and qPCR5, the following quasi-normalized masses (we use $\star$ symbol to specify the quasinormalization): 


\begin{tabular}{|l|ccccc|}
\hline & $\theta_{1}$ & $\theta_{2}$ & $\theta_{1} \cup \theta_{2}$ & $\emptyset$ & $\theta_{1} \cap \theta_{2}$ \\
\hline$q m_{1}()$. & $L_{1}$ & $L_{3}$ & $L_{1}$ & & \\
$q m_{2}()$. & $L_{2}$ & $L_{1}$ & $L_{2}$ & & \\
\hline$q m_{q D S m H}^{\star}()$. & $L_{2}$ & $L_{3}$ & $L_{3}$ & $L_{0}$ & $L_{0}$ \\
$q m_{q P C R 5}^{\star}()$. & $L_{3}$ & $L_{4}$ & $L_{0}$ & $L_{0}$ & $L_{0}$ \\
\hline
\end{tabular}

Table 10: Fusion with quasi-normalization

\section{Conclusion}

In this paper we have presented the foundations of DSmT and its main combination rules for dealing with both the quantitative or qualitative beliefs. The combination of qualitative beliefs published here results from very recent research investigations. DSmT although not sufficiently known in the information fusion and artificial intelligence communities as any new emerging theory has however already been successfully applied in different fields like multitarget tracking and classification, or remote sensing application. We hope that this special issue of Information \& Security Journal devoted to Fusing Uncertain, Imprecise and Conflicting information will help readers involved in information fusion to become curious and hopefully more comfortable with our research works and our new ideas in data fusion. DSmT is a new promising paradigm shift for the combination of precise (and even imprecise), uncertain and potentially highly conflicting quantitative or qualitative sources of information. It is important to emphasize that most of methods, like discounting techniques for example, developed to improve the management of quantitative beliefs in Dempster-Shafer Theory can also directly be applied in DSmT framework.

\section{References}

[1] Bobrow D., Qualitative reasoning about physical systems, Elsevier Publishers Ltd., North-Holland, 1984.

[2] Bolanos J., De Campos L.M., Moral S., Propagation of linguistic labels in causal networks, Proc. of 2nd IEEE Int. Conf. on Fuzzy Systems, Vol. 2, pp. 863-870, 28 March-1 April 1993.

[3] Bryson N., Mobolurin A.A., A qualitative discriminant approach for generating quantitative belief functions, IEEE Transactions on Knowledge and Data Engineering, Vol. 10, no. 2, pp. 345 - 348, March-April 1998.

[4] Darwiche A., Ginsberg M.L.,A Symbolic Generalization of Probability Theory, Proceedings of the Tenth National Conference on Artificial Intelligence, AAAI Press, Menlo Park, CA, Paul Rosenbloom and Peter Szolovits (Editors), pp. 622627, 1992. 
[5] Dezert J., Smarandache F., Daniel M., The Generalized Pignistic Transform, Proc. of Fusion 2004 Conf., Stockholm, July 2004.

[6] Dezert J., Smarandache F., A short overview on DSmT for Information Fusion, 10th Int. Conf. on Fuzzy Theory and Techn., Salt Lake City, Utah, USA, July 21-26, 2005.

[7] Dezert J., Smarandache F., The DSmT Approach for Information Fusion and Some Open Challenging Problems, NATO ASI, Albena, Bulgaria, 16-27 May 2005.

[8] Dezert J., Smarandache F., DSmT: A new paradigm shift for information fusion, Proceedings of Cogis'06 conference, 15-17 March 2006, Paris, France.

[9] Dezert J., Tchamova A., Smarandache F., Konstantinova P., Target Type Tracking with PCR5 and Dempster's rules - A Comparative Analysis, To appear in Proceedings of Fusion 2006 conference, 11-13 July 2006, Firenze, Italy.

[10] Dubois D., Prade H., On the unicity of Dempster rule of combination, Int. J. of Intelligent Syst., Vol. 1, pp 133-142, 1986.

[11] Dubois D., Prade H., Fuzzy arithmetic in qualitative reasoning, Modelling and Control of Systems in Engineering, Quantum Mechanics, Economics and Biosciences (Proc. of the Bellman Continuum Workshop 1988 Sophia Antipolis, p.457), Ed. Blaquière, A., Springer-Verlag, Berlin, 1989.

[12] Ferson S., Donald S., Probability bounds analysis, International Conference on Probabilistic Safety Assessment and Management (PSAM4), New York, NY, Springer-Verlag, 1998.

[13] Florea M. C., Dezert J., Valin P., Smarandache F., Anne-Laure Jousselme, Adaptative combination rule and proportional conflict redistribution rule for information fusion, Proc. of Cogis 2006 Conference, Paris, March 2006.

[14] Gagnon M.L., Rapport Fusion de Données, Technical Report, Département de Génie Electrique et de Génie informatique, Université de Laval, Canada, Novembre 2005.

[15] Haenni R., Shedding New Light on Zadeh's Criticism of Dempsters Rule of Combination, Proceedings of Fusion 2005 Conf., Philadelphia, July 26-29, 2005.

[16] Inagaki T., Interdependence between safety-control policy and multiple-sensor schemes via Dempster-Shafer theory, IEEE Trans. on reliability, Vol. 40, no. 2, pp. 182-188, 1991. 
[17] Lamata M. T., Moral S., Calculus with linguistic probabilities and beliefs, Chap. 7, pp. 133-152 in Advances In The Dempster-Shafer Theory of Evidence (Yager R., Kacprzyk J. and Fedrizzi M. Editors), John Wiley \& Sons, New York, 1994.

[18] Lefèvre E., Colot O., Vannoorenberghe P., Belief functions combination and conflict management, Inf. Fusion J., Vol. 3, No. 2, pp. 149-162, 2002.

[19] Martin A., Osswald C., A new generalization of the proportional conflict redistribution rule stable in terms of decision, to appear in Advances and Applications of DSmT for Information Fusion, (Collected Works, Vol. 2), F. Smarandache and J. Dezert (Editors), American Research Press (in preparation), July 2006.

[20] Ngwenyama O.K., Bryson N., Generating belief functions from qualitative preferences: an approach to eliciting expert judgments and deriving probability functions, Data \& Knowledge Eng., Vol. 28, no. 2, pp. 145-159, Nov. 1998.

[21] Parsons S., Mamdani E., Qualitative Dempster-Shafer Theory, Proceedings of the Third EMACS International Workshop on Qualitative Reasoning and Decision Technologies, Barcelona, Spain, 1993.

[22] Parsons S., Some qualitative approaches to applying Dempster-Shafer theory, Information and Decision technologies, Vol. 19, pp. 321-337, 1994.

[23] Parsons S., Hunter A., A review of uncertainty handling formalisms, Applications of Uncertainty Formalisms, pp. 8-37, 1998.

[24] Parsons S., A proof theoretic approach to qualitative probabilistic reasoning, Int. J. of Approx. Reas., Vol. 19, nos 3-4, pp. 265-297, Oct.-Nov. 1998.

[25] Parsons S., Qualitative Methods for Reasoning under Uncertainty, MIT Press, 2001.

[26] Parsons S., On precise and correct qualitative probabilistic inference, Int. J. of Approx. Reas., Vol. 35, no. 2, pp. 111-135, Feb. 2004.

[27] Polya G.,Patterns of Plausible Inference, Princeton University Press, Princeton, NJ, 1954.

[28] Sentz K., Ferson S., Combination of evidence in Dempster-Shafer Theory, SANDIA Tech. Report, SAND2002-0835, 96 pages, April 2002.

[29] Shafer G., A Mathematical Theory of Evidence, Princeton Univ. Press, Princeton, NJ, 1976.

[30] Smarandache F., Dezert J. (Editors), Applications and Advances of DSmT for Information Fusion, American Research Press, Rehoboth, 2004, http://www.gallup.unm.edu/ smarandache/DSmT-book1.pdf. 
[31] Smarandache F., Dezert J., Proportional Conflict Redistribution Rules for Information Fusion, to appear in Advances and Applications of DSmT for Information Fusion, (Collected Works, Vol. 2), F. Smarandache and J. Dezert (Editors), American Research Press (in preparation), July 2006 (preliminary version available at http://arxiv.org/pdf/cs.AI/0408064).

[32] Smarandache F., Dezert J., Information Fusion Based on New Proportional Conflict Redistribution Rules, Proceedings of Fusion 2005 Conf., Philadelphia, July 26-29, 2005.

[33] Smarandache F., Dezert J., A Simple Proportional Conflict Redistribution Rule, International Journal of Applied Mathematics \& Statistics, J. Mazumdar (Editor), ISSN 0973-1377, Vol.3, No. J05, pp. 1-36, June 2005.

[34] Smarandache F., Dezert J., An introduction to DSm Theory of Plausible, Paradoxist, Uncertain, and Imprecise Reasoning for Information Fusion, WOSC 13th Int. Cong. of Cybernetics and Syst., Maribor, Slovenia, July 6-10, 2005.

[35] Smets Ph., Belieffunctions, Non-standard logics for automated reasoning, Smets, Mamdani, Dubois and Prade Editors, Academic Press, 1988.

[36] Smets Ph., Kennes R., The transferable Belief Model, Artif. Intel., Vol. 66, no. 2, pp. 191-234, 1994.

[37] Voorbraak F., On the justification of Dempster's rule of combination, Artificial Intelligence, 48, pp. 171-197, 1991.

[38] Walley P., Statistical Reasoning with Imprecise Probabilities, New York, Chapman and Hall, 1991.

[39] Wellman M.P., Some varieties of qualitative probability, Proc. of the 5th Int. Conf. on Information Processing and the Management of Uncertainty, IPMU 1994, Paris, July 1994.

[40] Wong S.K.M., Yao, Y.Y., Bollmann-Sdorra P., Qualitative fuzzy system structures, Proc. of 2nd IEEE Int. Conf. on Fuzzy Syst., Vol. 2, pp. 857-862, 28 March-1 April 1993.

[41] Wong S.K.M., Lingras P., Representation of qualitative user preference by quantitative belief functions IEEE Transactions on Knowledge and Data Engineering, Vol. 6, no. 1, pp. 72-78, Feb. 1994.

[42] Yager R.R., On the Dempster-Shafer framework and new combination rules, Inf. Sci., Vol. 41, pp. 93-138, 1987. 
[43] Yager R. R., On the retranslation process in Zadeh's paradigm of computing with words, IEEE Trans. on Systems, Man, and Cybernetics - Part B: Cybernetics, Vol. 34, no. 2, pp. 1184-1195, April 2004.

[44] Zadeh L., Concept of a linguistic variable and its application to approximate reasoning, Information Sciences, Part 1, Vol. 8, pp. 199-249, 1975.

[45] Zadeh L., A Theory of Approximate Reasoning, Machine Intelligence, Vol. 9, pp. 149-194, 1979.

[46] Zadeh L., On the validity of Dempster's rule of combination, Memo M 79/24, Univ. of California, Berkeley, 1979.

[47] Zadeh L., A simple view of the Dempster-Shafer theory of evidence and its implication for the rule of combination, AI Magazine 7, No.2, pp. 85-90, 1986.

[48] Zadeh L., Fuzzy Logic = Computing with Words, IEEE Transactions on Fuzzy Systems, Vol. 4, no. 2, pp. 103-111, May 1996.

[49] Zadeh L., Towards a theory of fuzzy information granulation and its centrality in human reasoning and fuzzy logic, Fuzzy Sets and Systems, Vol. 19, pp. 111-127, 1997.

[50] Zadeh L., Some reflections on soft computing, granular computing and their roles in the conception, design and utilization of information/intelligent systems, Soft Computing, Vol. 2., pp. 23-25, 1998.

[51] Zadeh L., Precisiated Natural Language - Toward a radical enlargement of the role of natural languages in Information Processing, Decision and Control, Proceedings of the 9th Int. Conf. on Neural Information Processing (ICONIP'02), Vol.1, pp. 1-3, Lipo Wang, Jagath C. Rajapakse, Kunihiko Fukushima, SooYoung Lee and Xin Yao (Editors), 2002. 
JEAN DEZERTwas born in l'Hay les Roses, France, on August 25, 1962. He received the electrical engineering degree from the Ecole Française de Radio-électricité et d'Electronique and Informatique (EFREI), Paris, in 1985, the D.E.A. degree in 1986 from the University Paris VII (Jussieu), and his Ph.D. from the University Paris XI, Orsay, in 1990, all in Automatic Control and Signal Processing. During 1986-1990 he was with the Systems Department at the Office National d'Etudes et de Recherches Aérospatiales (ONERA), Châtillon, France, and did research in tracking. During 1991-1992, he visited the Department of Electrical and Systems Engineering, University of Connecticut, Storrs, U.S.A. as an European Space Agency (ESA) Postdoctoral Research Fellow. During 1992-1993 he was teaching assistant in Electrical Engineering at the University of Orléans, France. Since 1993, he is senior research scientist in the Image Estimation and Decision (IED) Research Lab. with the Information and Modelling and Processing Department (DTIM) at ONERA. His current research interests include autonomous navigation, estimation theory, stochastic systems theory and its applications to multisensor-multitarget tracking (MS-MTT), information fusion, plausible reasoning and non-standard Logics. Dr. Jean Dezert is developing since 2001 with Professor Smarandache a new theory of plausible and paradoxical reasoning for information fusion (DSmT) and has edited the first textbook (collected works) devoted to this new emerging research field published by American Research Press, Rehoboth in 2004. The second volume is under preparation and will be in print in June 2006. He owns one international patent in the autonomous navigation field and has published several papers in international conferences and journals. He coauthored a chapter in Multitarget-Multisensor Tracking: Applications and Advances, Vol.2 (Y. Bar-Shalom Editor). He is member of IEEE and of Eta Kappa Nu, serves as reviewer for different International Journals, teaches a MS-MTT and Data Fusion course at the French ENSTA Engineering School, collaborates for the development of the International Society of Information Fusion (ISIF) since 1998, and has served as Local Arrangements Organizer for the Third International Conference on Information Fusion, FUSION 2000, July 10- 13, in Paris. He has been involved in the Technical Program Committees of Fusion 2001-2004 International Conferences. Since 2001, he is a member of the board of the International Society of Information Fusion (http://www.isif.org) and served as secretary for ISIF since 2001. He served as executive vice-president of ISIF in 2004. In 2003, he organized with Professor Smarandache, the first special session devoted to plausible and paradoxical reasoning for information fusion at the International conference on Information Fusion, Fusion 2003, Cairns, Australia and also a panel discussion and a special session on DSmT at Fusion 2004, Stockholm in June 2004. Dr. Dezert gave several invited seminars and lectures on Data Fusion and Tracking during recent past years. He also participates as member to Conference Technical Committee of Fusion 2005, Fusion 2006 International Conference on Information Fusion and Fuzzy Sets and Technology Conference, Salt Lake City, USA in July 2005. He is also Associate Editor of Journal of Advances in Information Fusion (JAIF). Most recent advances on DSmT can be found at : http://www.gallup.unm.edu/ smarandache/DSmT.htm

FLORENTIN SMARANDACHE Florentin Smarandache was born in Balcesti, Romania, in 1954. He got a M. Sc. Degree in both Mathematics and Computer Science from the University of Craiova in 1979, received a Ph. D. in Mathematics from the State University of Kishinev in 1997, and continued postdoctoral studies at various American Universities ( New Mexico State University in Las Cruces, Los Alamos National Laboratory) after emigration. In 1988 he escaped from his country, pasted two years in a political refugee camp in Turkey, and in 1990 emigrated 
to USA. In 1996 he became an American citizen. Dr. Smarandache worked as a professor of mathematics for many years in Romania, Morocco, and United States, and between 1990-1995 as a software engineer for Honeywell, Inc., in Phoenix, Arizona. In present, he teaches mathematics at the University of New Mexico, Gallup Campus. Very prolific, he is the author, coauthor, and editor of 75 books, over 100 scientific notes and articles, and contributed to about 50 scientific and 100 literary journals from around the world (in mathematics, informatics, physics, philosophy, rebus, literature, and arts). He wrote in Romanian, French, and English. Some of his work was translated into Spanish, German, Portuguese, Italian, Dutch, Arabic, Esperanto, Swedish, Farsi, Arabic, Chinese. He was so attracted by contradictions that, in 1980s, he set up the "Paradoxism" avant-garde movement in literature, philosophy, art, even science, which made many advocates in the world, and it's based on excessive use of antitheses, antinomies, paradoxes in creation - making an interesting connection between mathematics, engineering, philosophy, and literature [http://www.geocities.com/charlestle/paradoxism.html] and led him to coining the neutrosophic logic, a logic generalizing the intuitionistic fuzzy logic that is able to deal with paradoxes. In mathematics there are several entries named Smarandache Functions, Sequences, Constants, and especially Paradoxes in international journals and encyclopedias. He organized the 'First International Conference on Neutrosophics' at the University of New Mexico, 1-3 December 2001 [http://www.gallup.unm.edu/ smarandache/FirstNeutConf.htm]. Small contributions he had in physics and psychology too. Much of his work is held in "The Florentin Smarandache Papers" Special Collections at the Arizona State University, Tempe, and Texas State University, Austin (USA), also in the National Archives (Rm. Vlcea) and Romanian Literary Museum (Bucharest), and in the Muse de Bergerac (France). In 2003, he organized with Dr. Jean Dezert, the first special session devoted to plausible and paradoxical reasoning for information fusion at the Fusion 2003 International conference on Information Fusion in Cairns, Australia. 